Supporting Information for:

\title{
The Role of Backbone Polarity on Aggregation and Conduction of Ions in Polymer Electrolytes
}

Nicole S. Schauser, ${ }^{\mathrm{a}, \mathrm{b}}$ Douglas J. Grzetic, ${ }^{\mathrm{b}}$ Tarnuma Tabassum, ${ }^{\mathrm{c}}$ Gabrielle A. Kliegle, ${ }^{\mathrm{b}, \mathrm{c}}$ My Linh Le, ${ }^{\mathrm{d}}$ Ethan M. Susca, ${ }^{\mathrm{b}}$ Ségolène Antoine, ${ }^{\mathrm{b}}$ Timothy J. Keller, ${ }^{\mathrm{c}}$ Kris T. Delaney, Songi Han,c Ram Seshadri,a,b,c Glenn H. Fredrickson, a,b,d, ${ }^{*}$ Rachel A. Segalman ${ }^{\mathrm{a}, \mathrm{b}, \mathrm{d},{ }^{*}}$

${ }^{a}$ Materials Department, ${ }^{b}$ Materials Research Laboratory, ${ }^{\mathrm{c}}$ Department of Chemistry and Biochemistry, and ${ }^{\mathrm{d} D e p a r t m e n t}$ of Chemical Engineering, University of California, Santa Barbara, California 93106, United States

*Email: ghf@ucsb.edu, segalman@ucsb.edu 


\section{Molecular Characterization}

NMR and SEC were used for synthesis verification and molecular weight determination. For the PAGE backbone, NMR was used for molecular weight determination based on end group analysis, with 5 protons on the phenyl end group $(7.3 \mathrm{ppm})$ compared with one vinyl proton located at $5.8 \mathrm{ppm}$ (see Figure S1). Functionalization with imidazole was verified by NMR in DMSO-d6 with the disappearance of all vinyl functional character between 5 and $6 \mathrm{ppm}$ for all backbones (see Figures S1, S2 and S3).

$$
m=\text { number of repeat units }=\frac{I_{\text {phenyl }}}{5} * I_{\text {vinyl }}=\frac{1.0}{5} * 14.2=71
$$

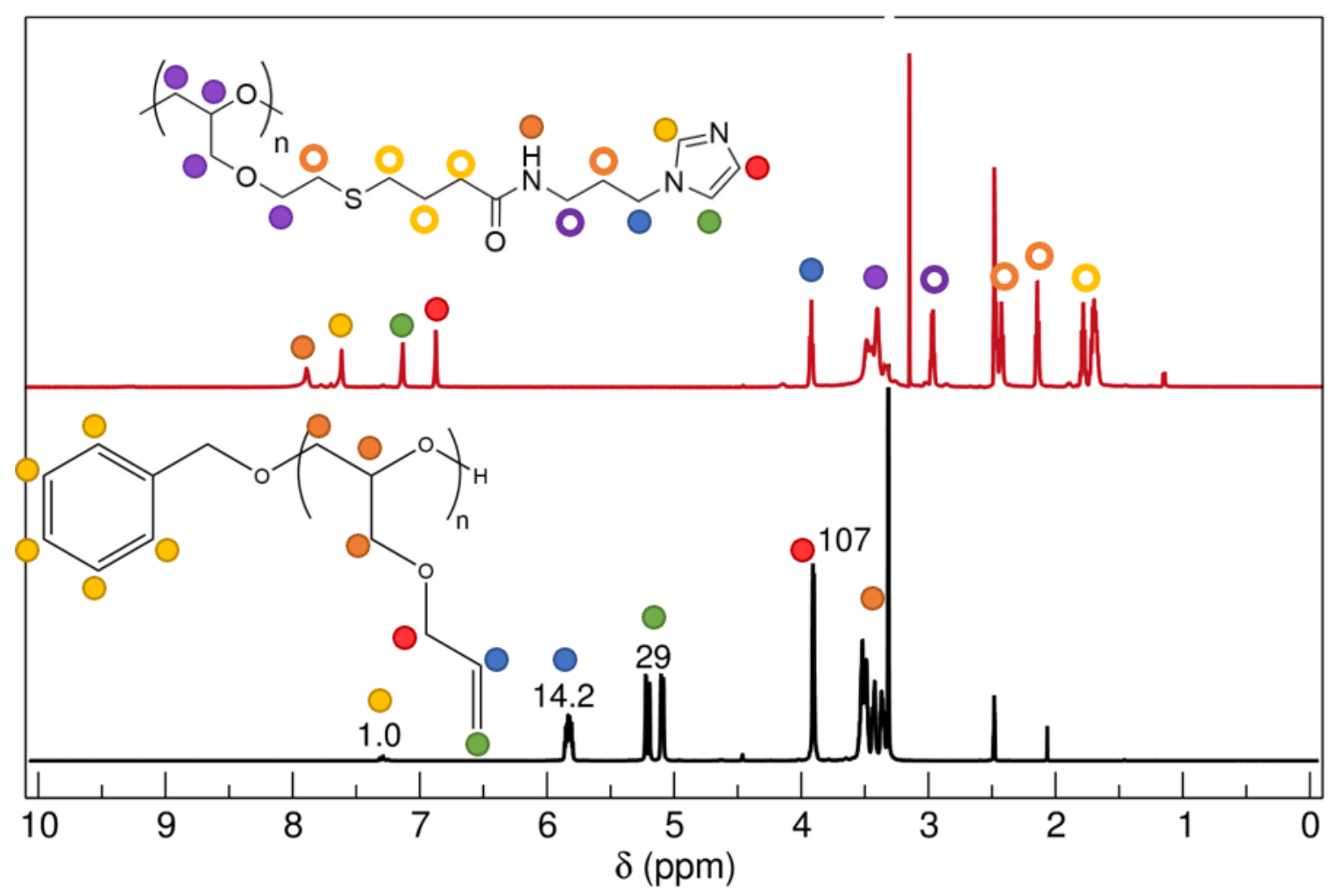

Figure S1. ${ }^{1} \mathrm{H}$ NMR of PAGE (black) and PAGE-Im in DMSO-d6. End group analysis of PAGE suggests 71 repeat units per chain. The disappearance of vinyl character suggests full functionalization for PAGE-Im. 


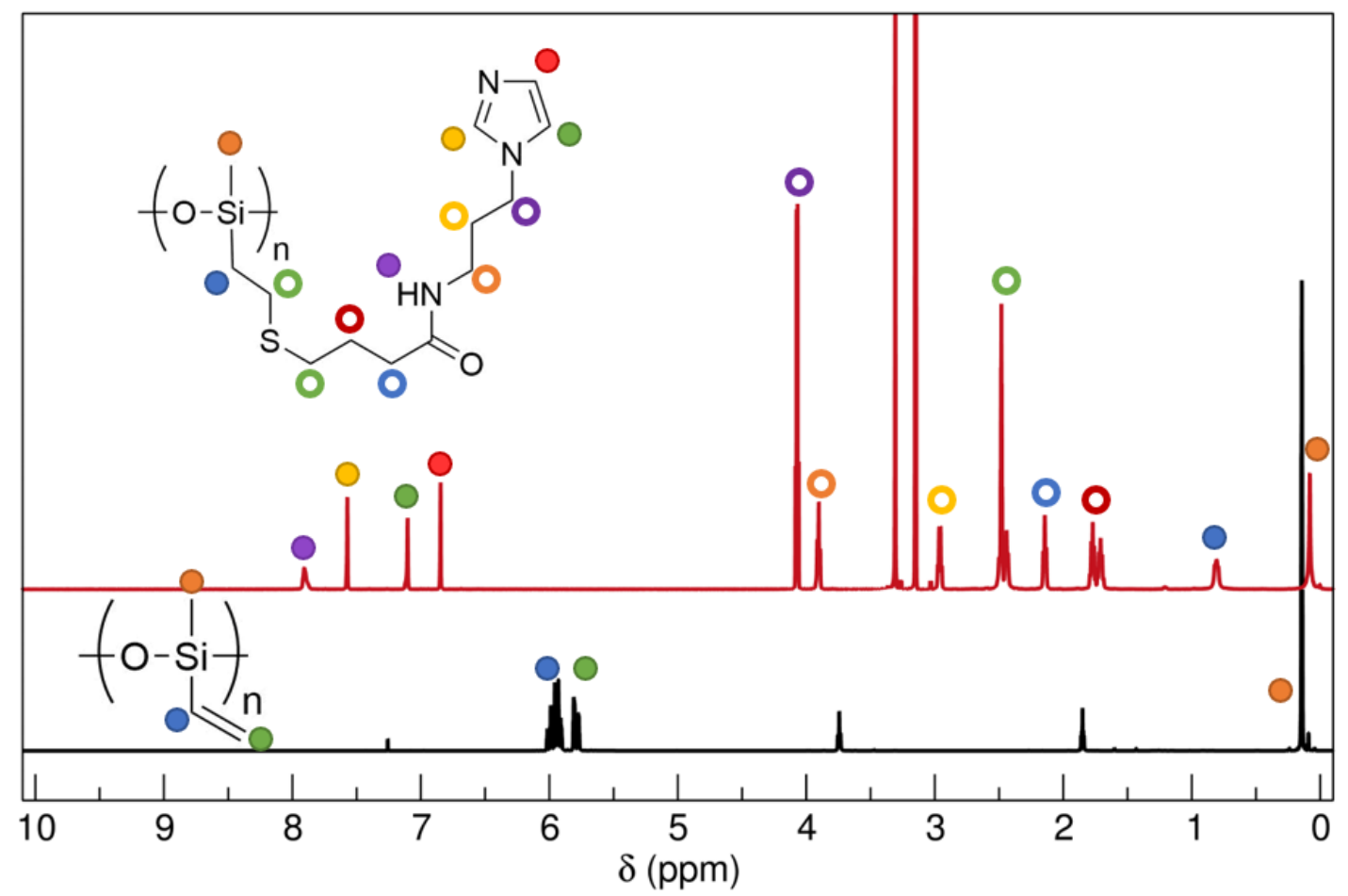

Figure S2. ${ }^{1} \mathrm{H}$ NMR of PVMS in $\mathrm{CDCl}_{3}$ (black) and PVMS-Im in DMSO-d6 (red) showing the disappearance of vinyl character upon functionalization and the appearance of the aromatic imidazole peaks between 6.5 and 8 ppm.

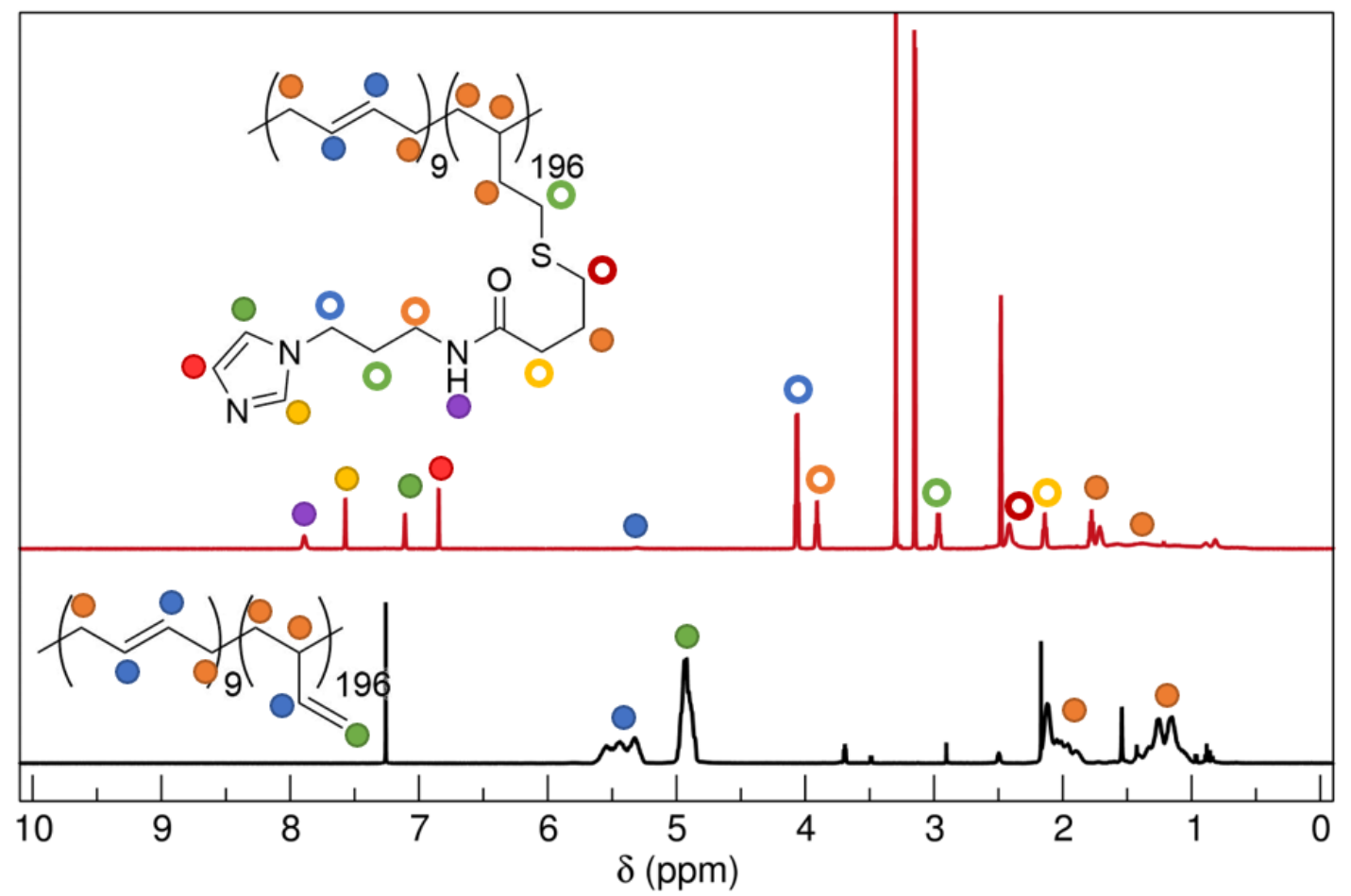

Figure S3. ${ }^{1} \mathrm{H}$ NMR of PBD in $\mathrm{CDCl}_{3}$ (black) and PBD-Im in DMSO-d6 (red), showing the disappearance of vinyl character (4.7-5.7 ppm) upon functionalization. 


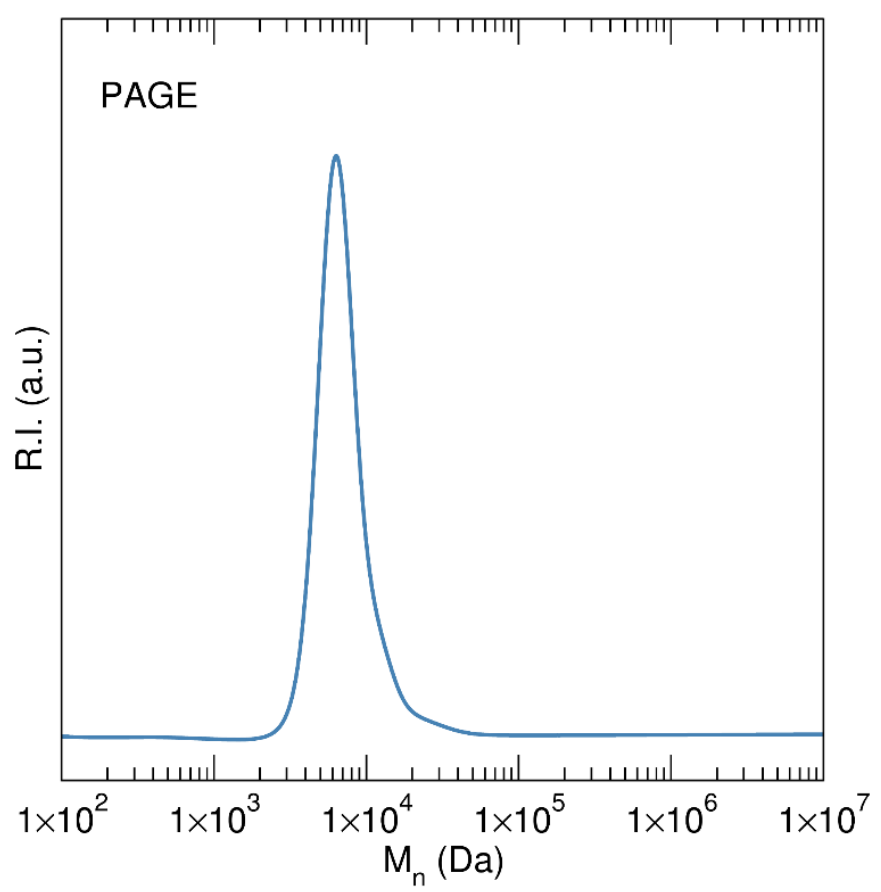

Figure S4. SEC trace of PAGE calibrated against polystyrene standards. The polydispersity was below 1.2 , with $M_{\mathrm{n}}=7.3 \mathrm{kDa}$.

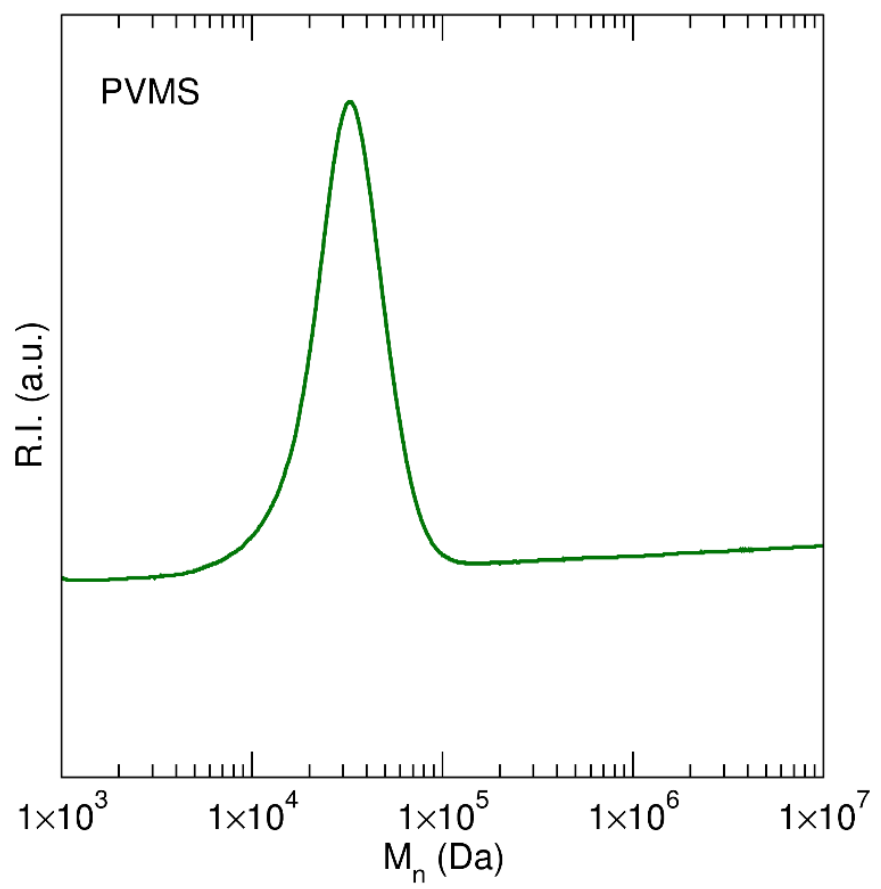

Figure S5. SEC trace of PVMS calibrated against polystyrene standards. The polydispersity was below 1.25 , with $M_{\mathrm{n}}=29.5 \mathrm{kDa}$. 


\section{Ionic Conductivity}

All conductivity samples were measured as a function of temperature and fit to VFT formalism as $\sigma=A \exp \left(\frac{-B}{T-T_{g}+50}\right)$. A representative conductivity plot is shown in Figure S6 for PAGE-Im with $\mathrm{Cu}(\mathrm{TFSI})_{2}$ at a molar ratio of 0.08 .

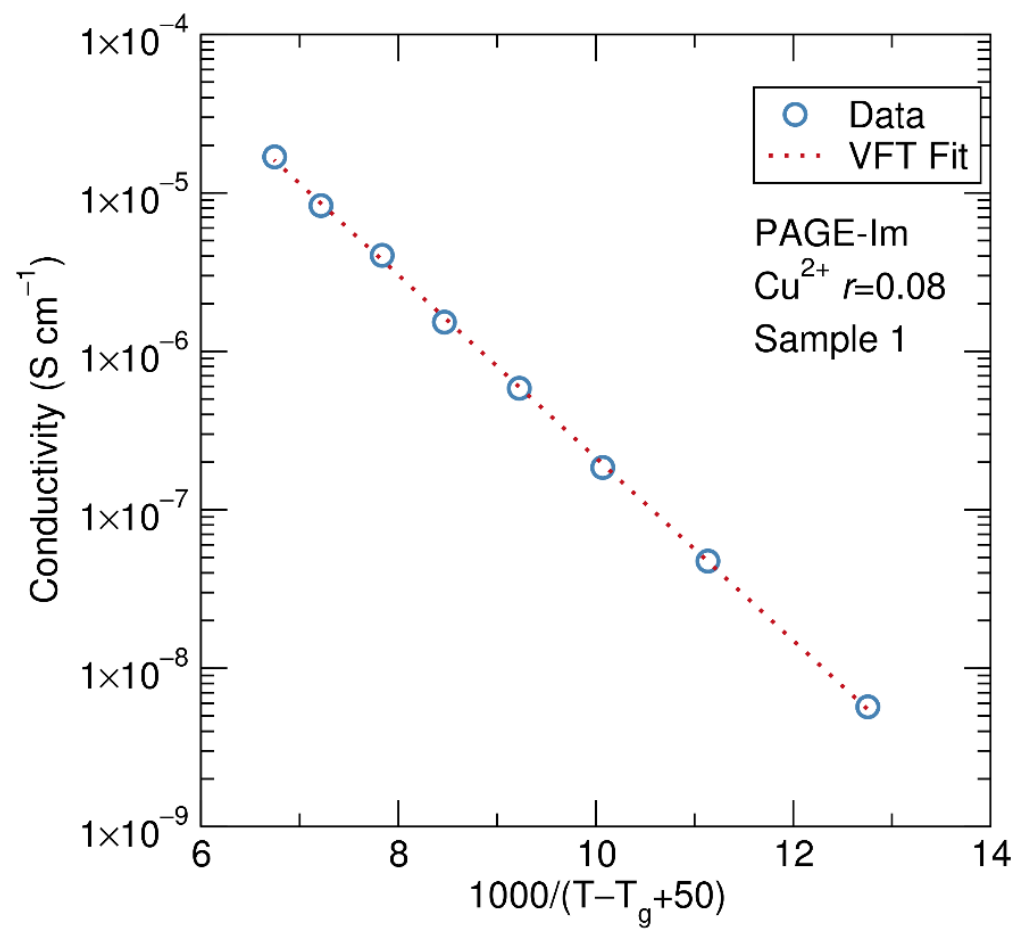

Figure S6. Ionic conductivity versus temperature adjusted by the VFT formalism for a representative sample, PAGE-Im with $\mathrm{Cu}(\mathrm{TFSI})_{2}$ at a metal to imidazole ratio of 0.08 . The VFT fit to the data is shown in the dotted line. 


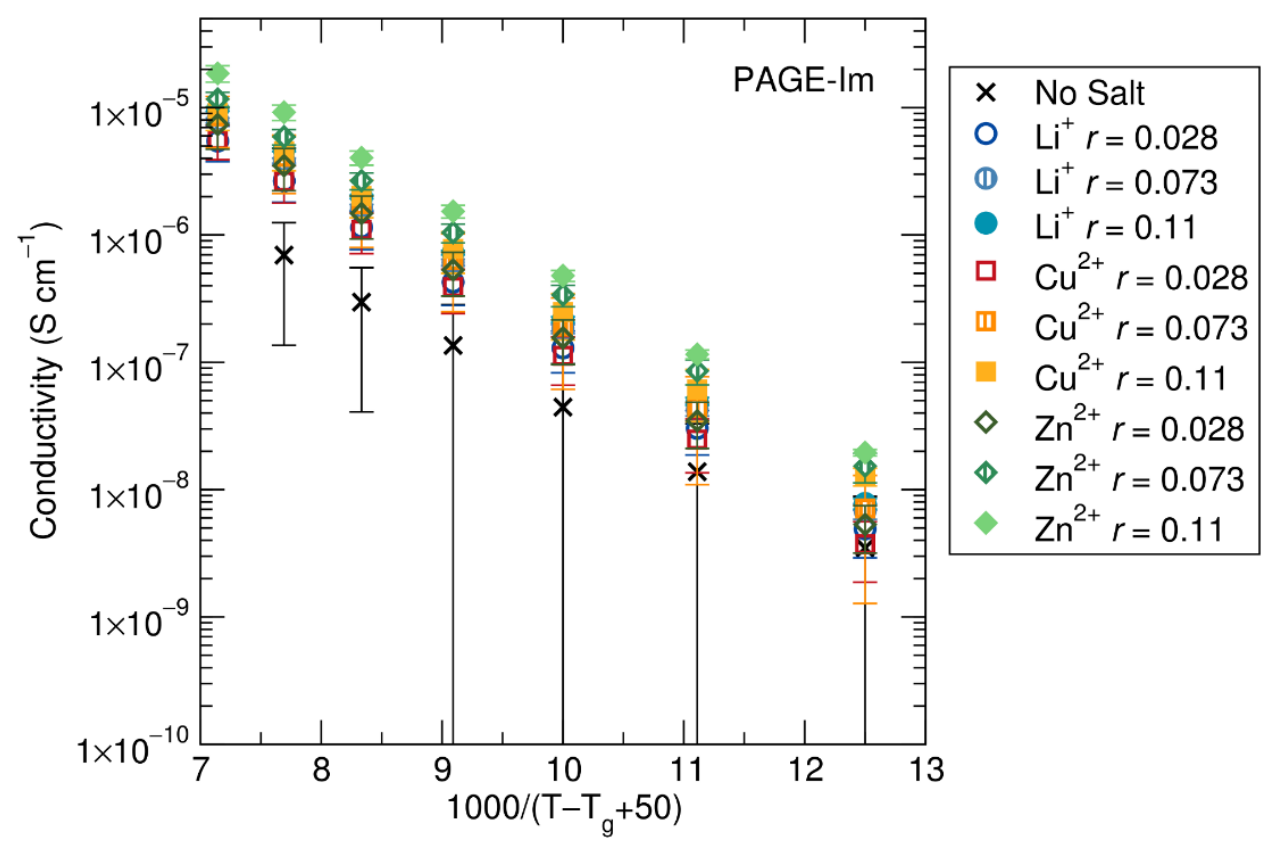

Figure S7. Average ionic conductivity versus temperature for PAGE-Im polymers. Error bars show standard deviation.

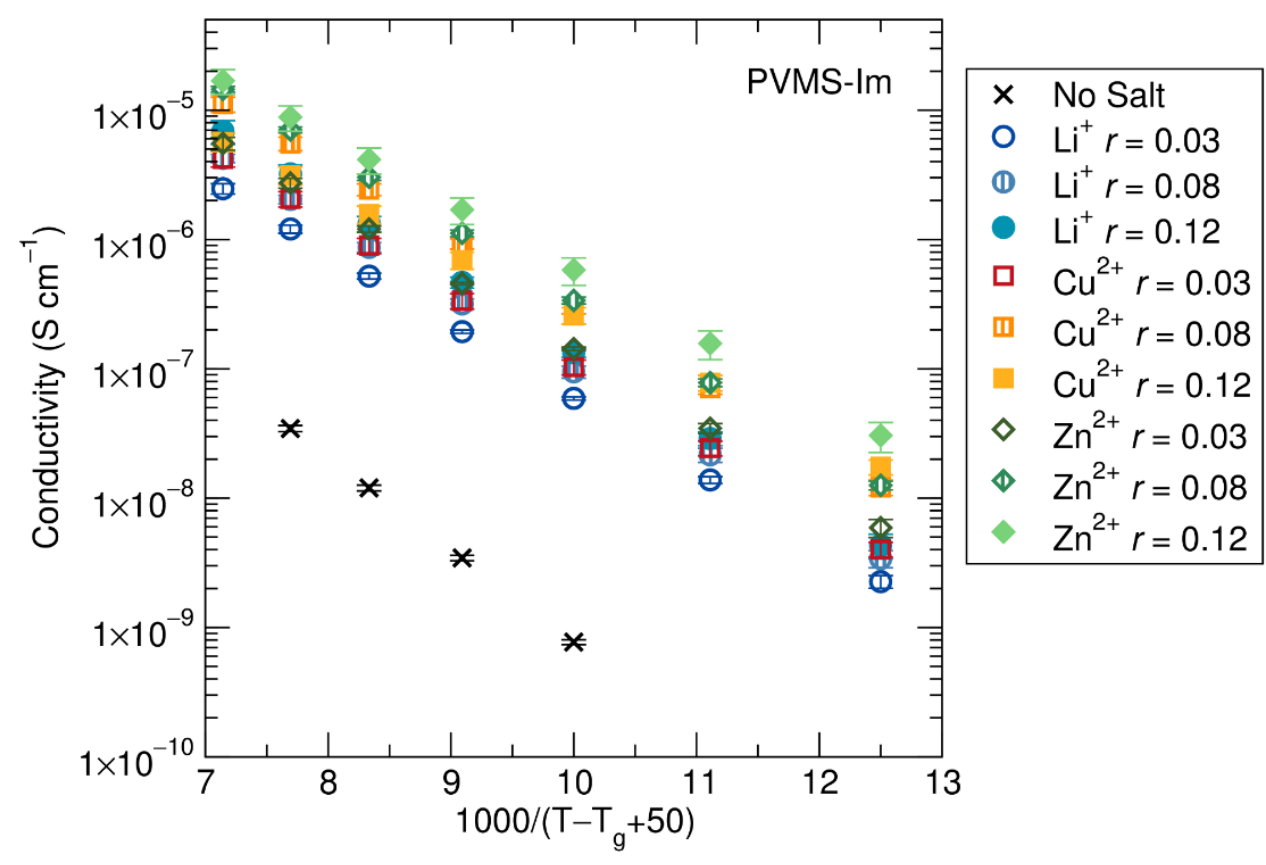

Figure S8. Average ionic conductivity versus temperature for PVMS-Im polymers. Error bars show standard deviation. 


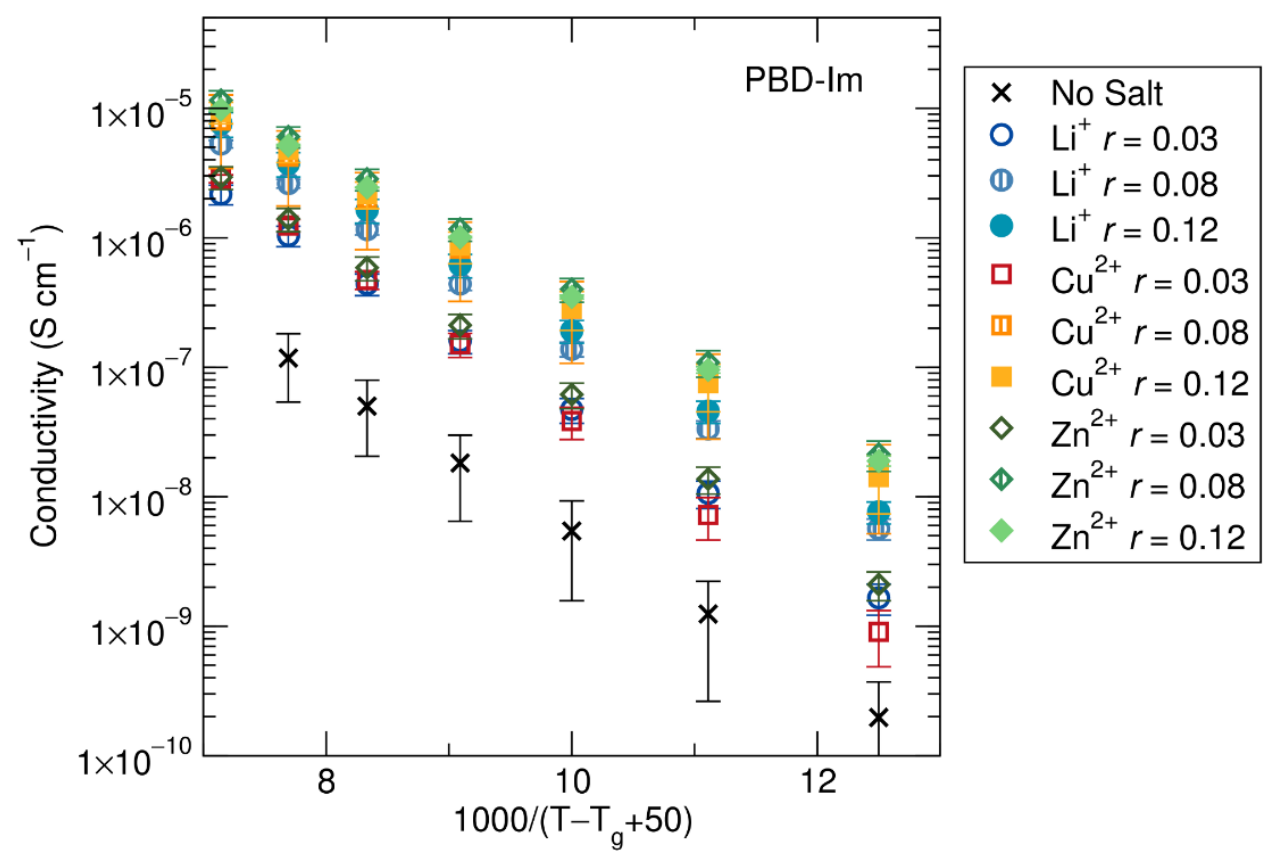

Figure S9. Average ionic conductivity versus temperature for PBD-Im polymers. Error bars show standard deviation.

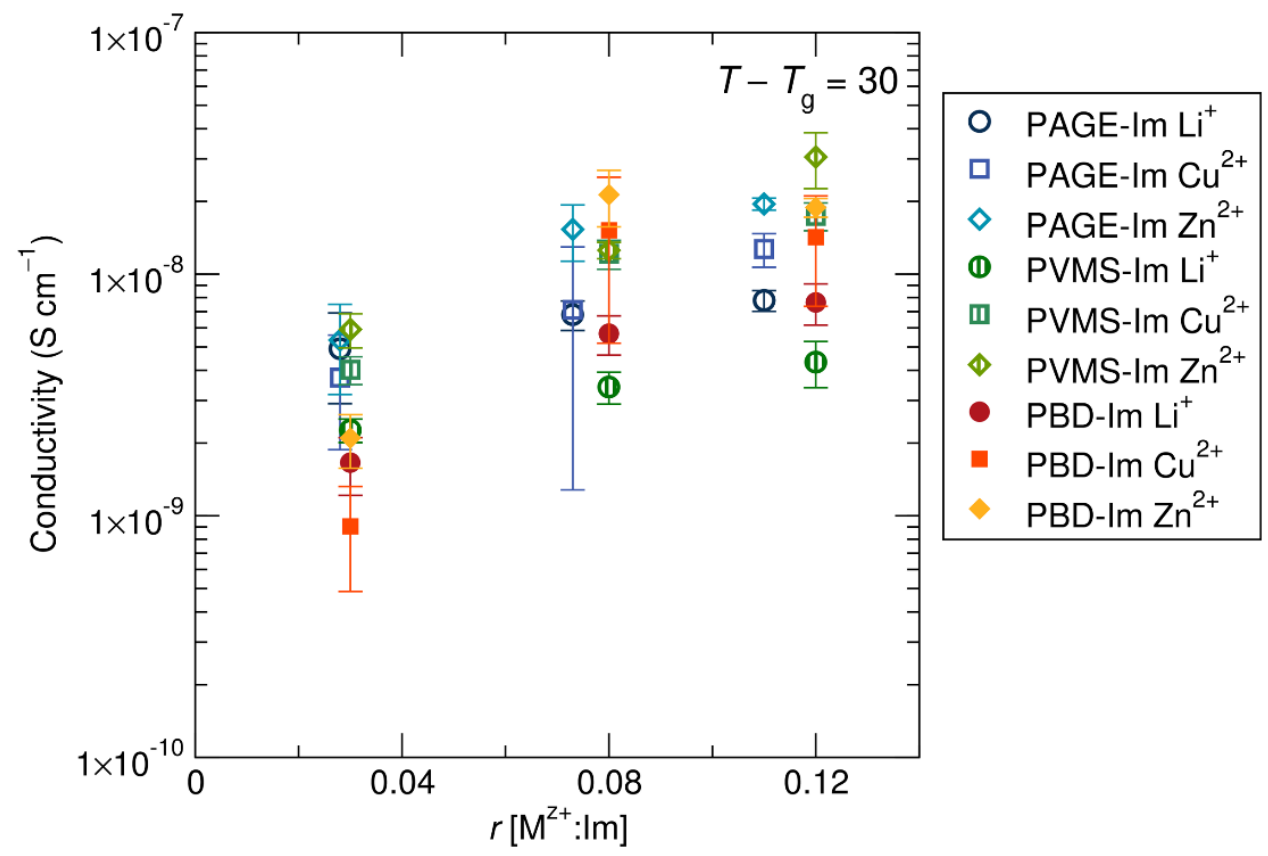

Figure S10. Ionic conductivity at $T-T_{\mathrm{g}}=30$ versus salt concentration. 


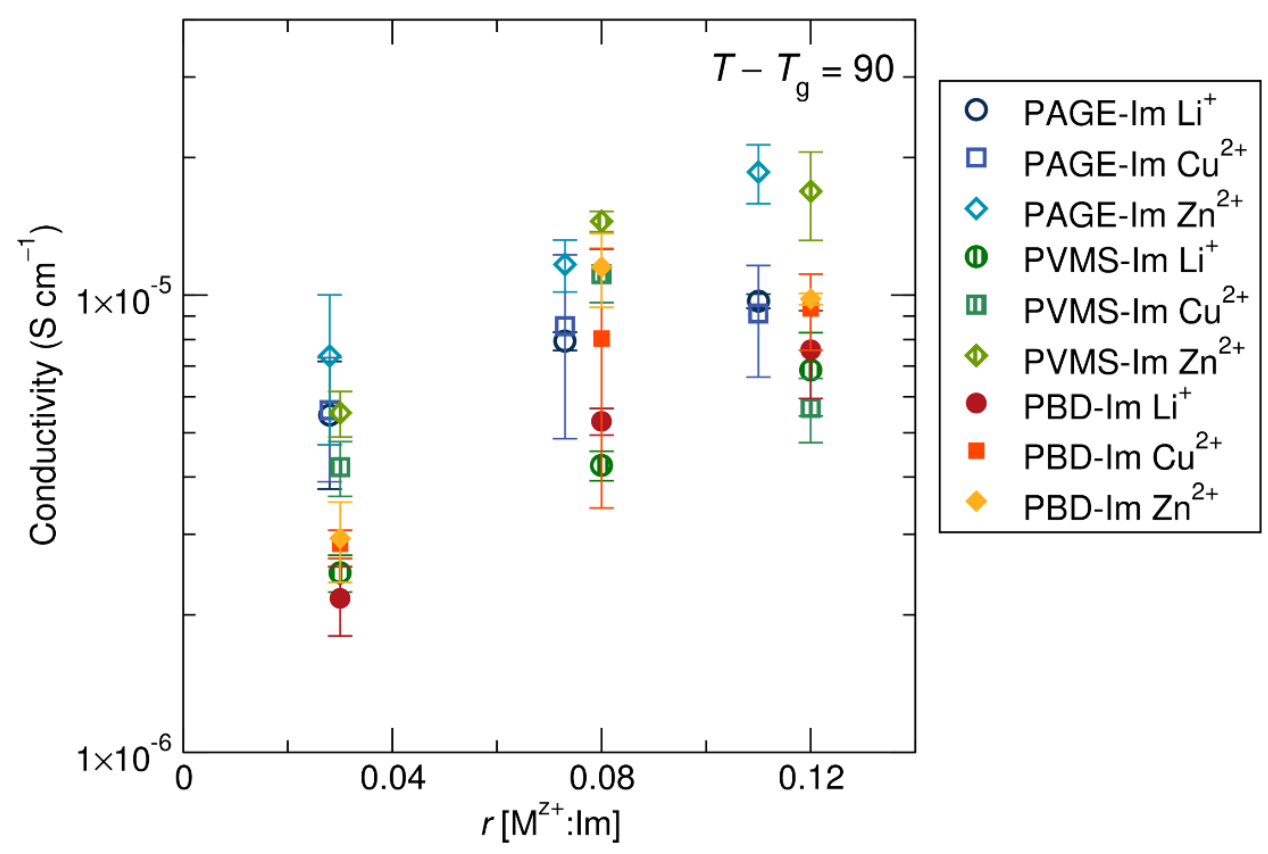

Figure S11. Ionic conductivity at $T-T_{\mathrm{g}}=90$ versus salt concentration.

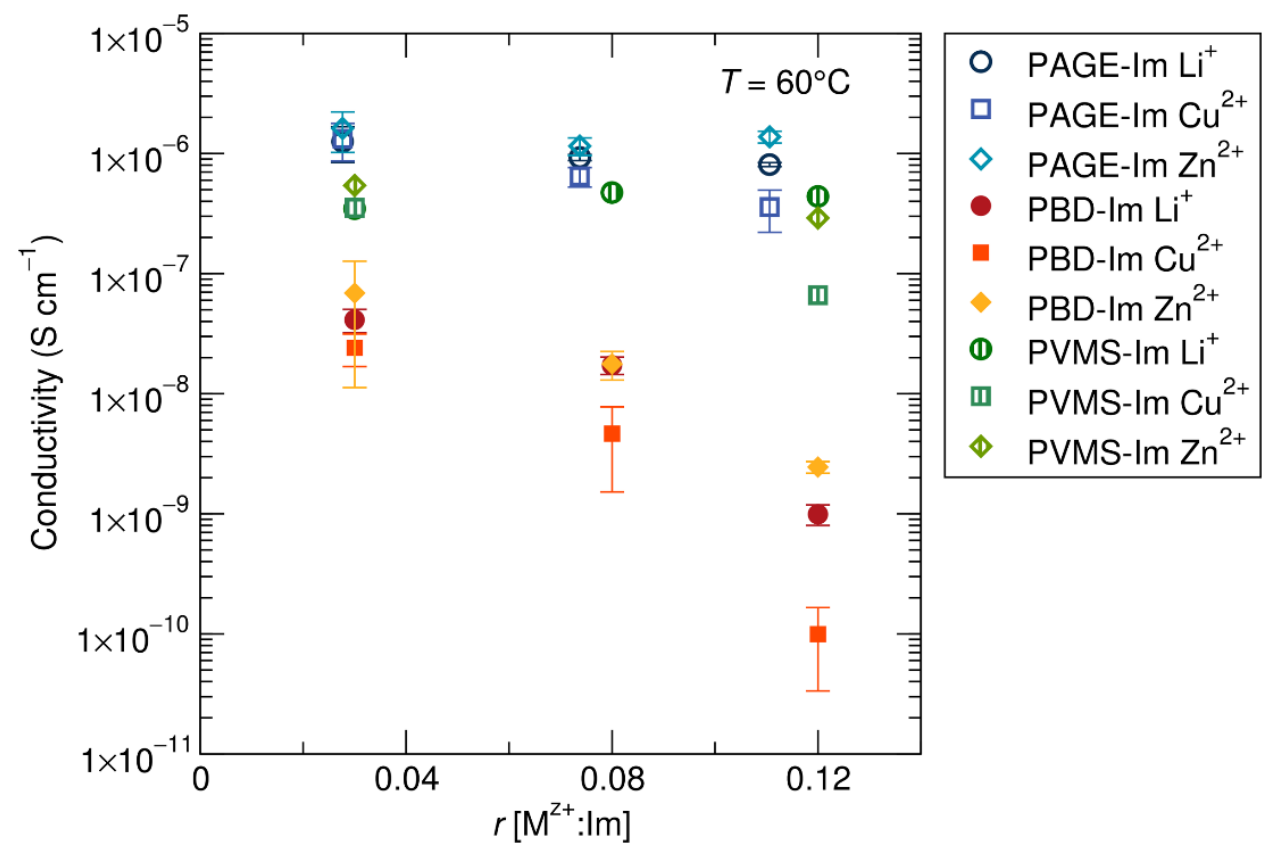

Figure S12. Ionic conductivity as a function of salt content at a constant temperature of $60{ }^{\circ} \mathrm{C}$. Conductivity decreases with increasing salt content due to an increase in $T_{\mathrm{g}}$. This is especially stark for the PBD backbone due to the proximity to $T_{\mathrm{g}}$, which reaches $50{ }^{\circ} \mathrm{C}$ for the PBD-Im $\mathrm{Cu}^{2+} r=0.12$ sample. 


\section{DSC Measurement}

All plots are shown upon second heating, endo up.
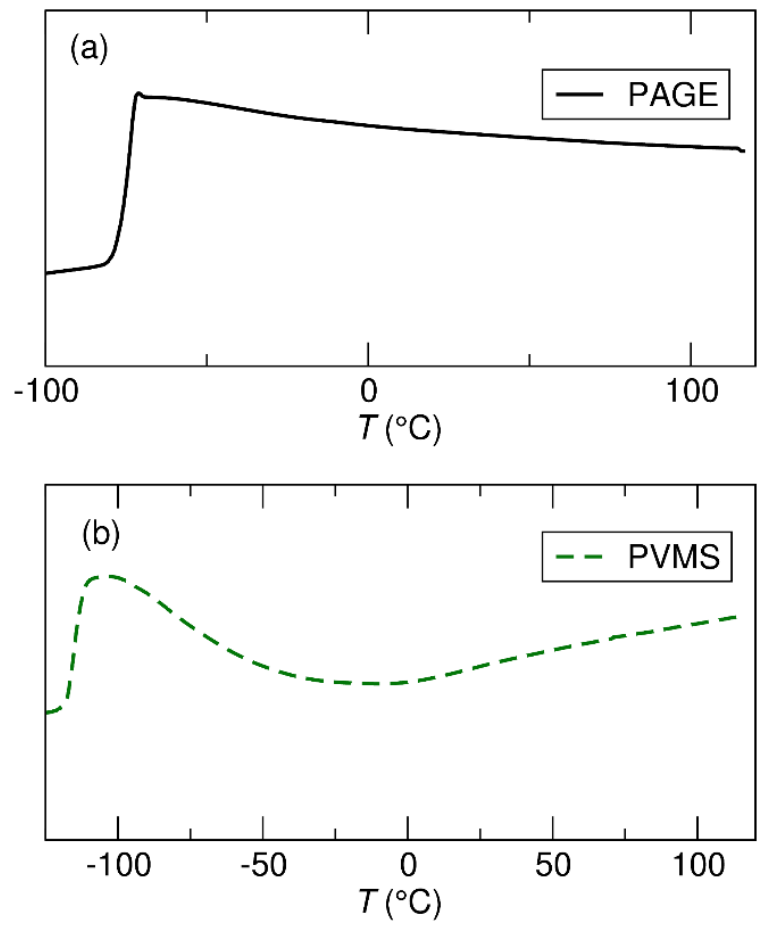

Figure S13. DSC data upon second heating of (a) PAGE and (b) PVMS before functionalization.

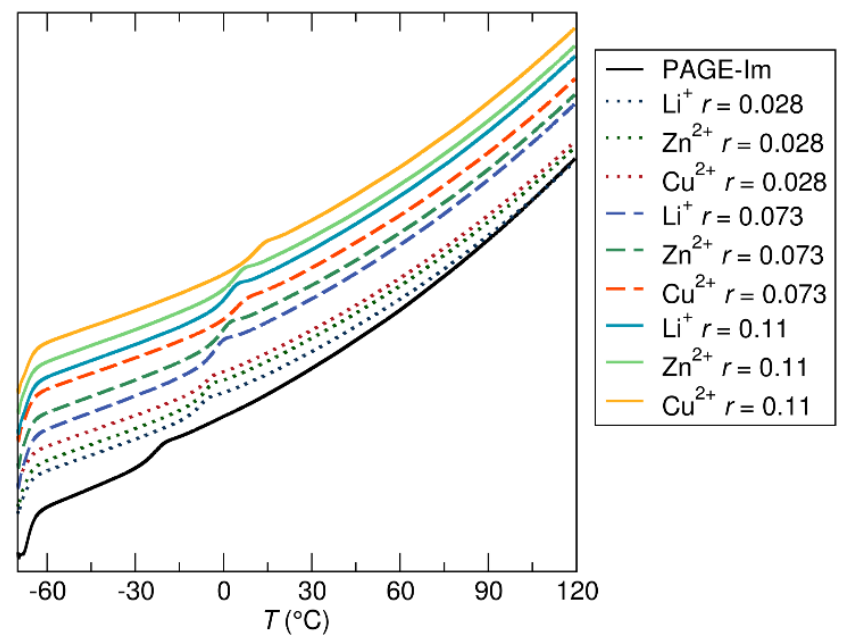

Figure S14. DSC data upon second heating of PAGE-Im with and without salt showing the shift in $T_{\mathrm{g}}$ with salt identity and concentration. 


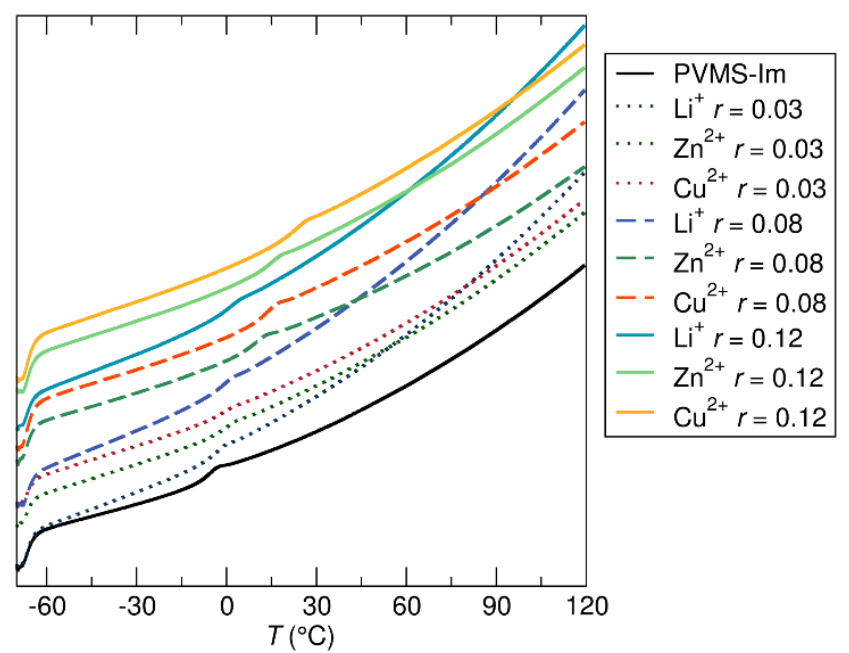

Figure S15. DSC data upon second heating of PVMS-Im with and without salt showing the shift in $T_{\mathrm{g}}$ with salt identity and concentration.

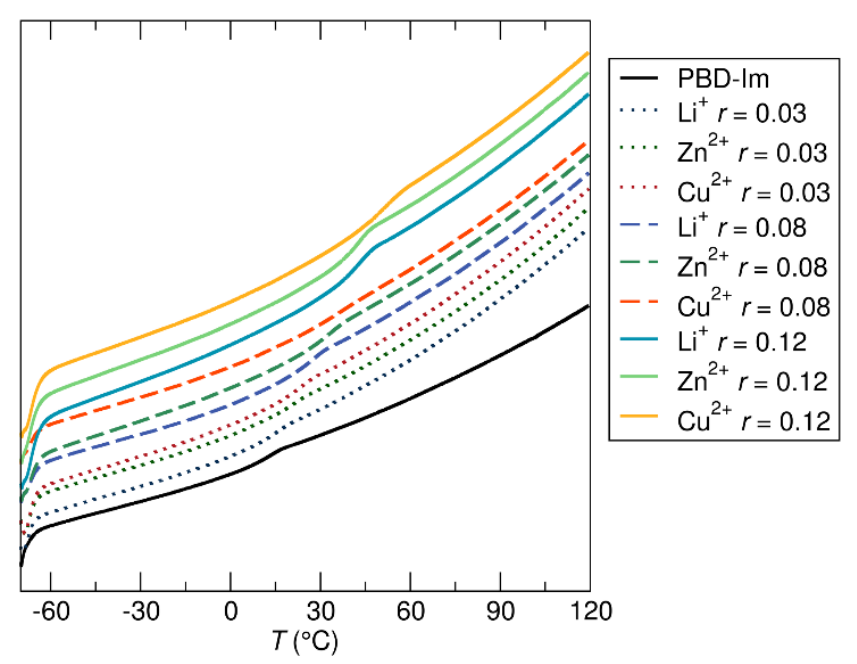

Figure S16. DSC data upon second heating of PBD-Im with and without salt showing the shift in $T_{\mathrm{g}}$ with salt identity and concentration. 


\section{Dielectric Constant Measurement}

Dielectric constants were measured at $-20{ }^{\circ} \mathrm{C}$ to minimize the onset of polarization which would lead to an overestimation of the dielectric constant. PAGE, PVMS and PBD were measured before and after functionalization, as shown in Figures S17 through S22. The dielectric constant is calculated from the sample capacitance divided by the cell constant, $C_{0}=\varepsilon_{0} A / t$. While reproducible noise is present in all samples, the value of the dielectric constant does not change significantly.

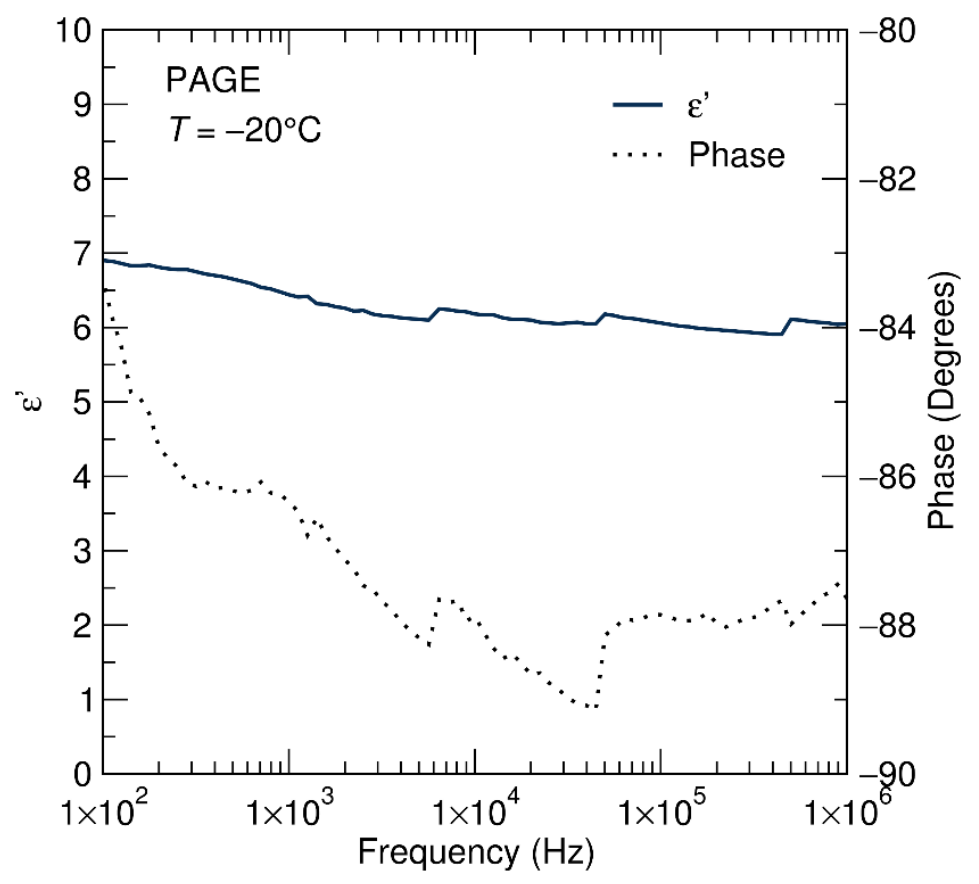

Figure S17. Dielectric constant and phase as a function of frequency for PAGE at $-20{ }^{\circ} \mathrm{C}$. Dielectric constants in Table 1 are reported at $1 \mathrm{MHz}$. 


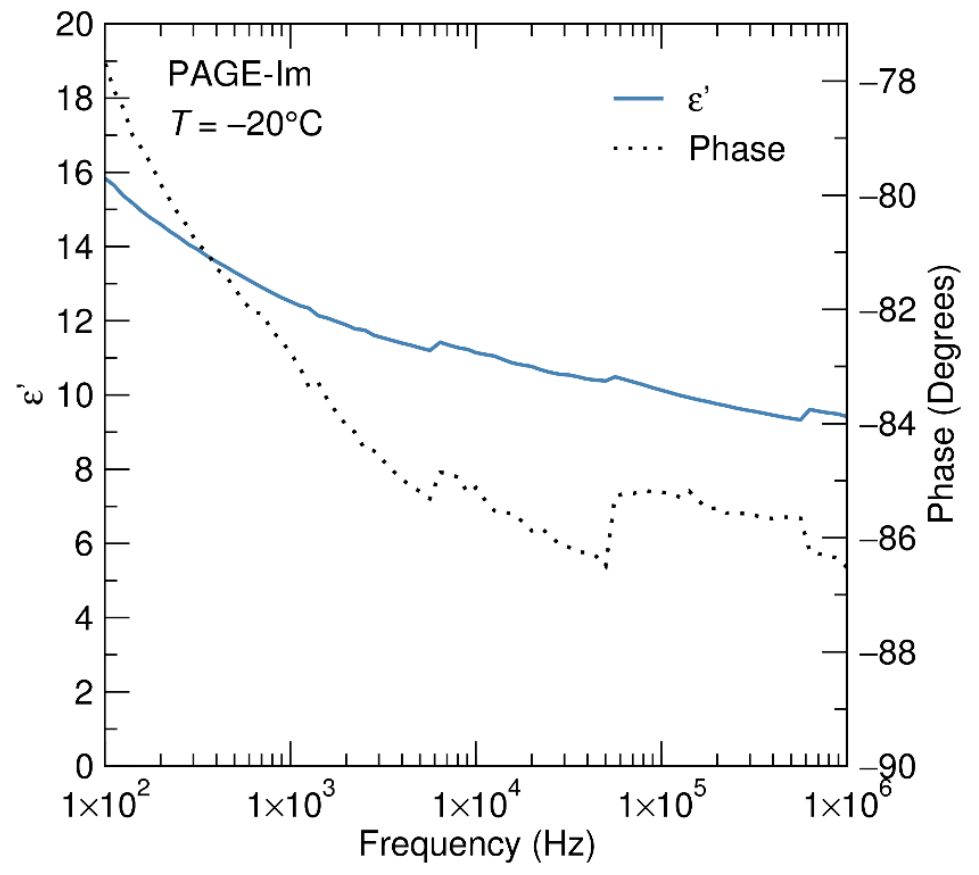

Figure S18. Dielectric constant and phase as a function of frequency for PAGE-Im at $-20{ }^{\circ} \mathrm{C}$. Dielectric constants in Table 1 are reported at $1 \mathrm{MHz}$.

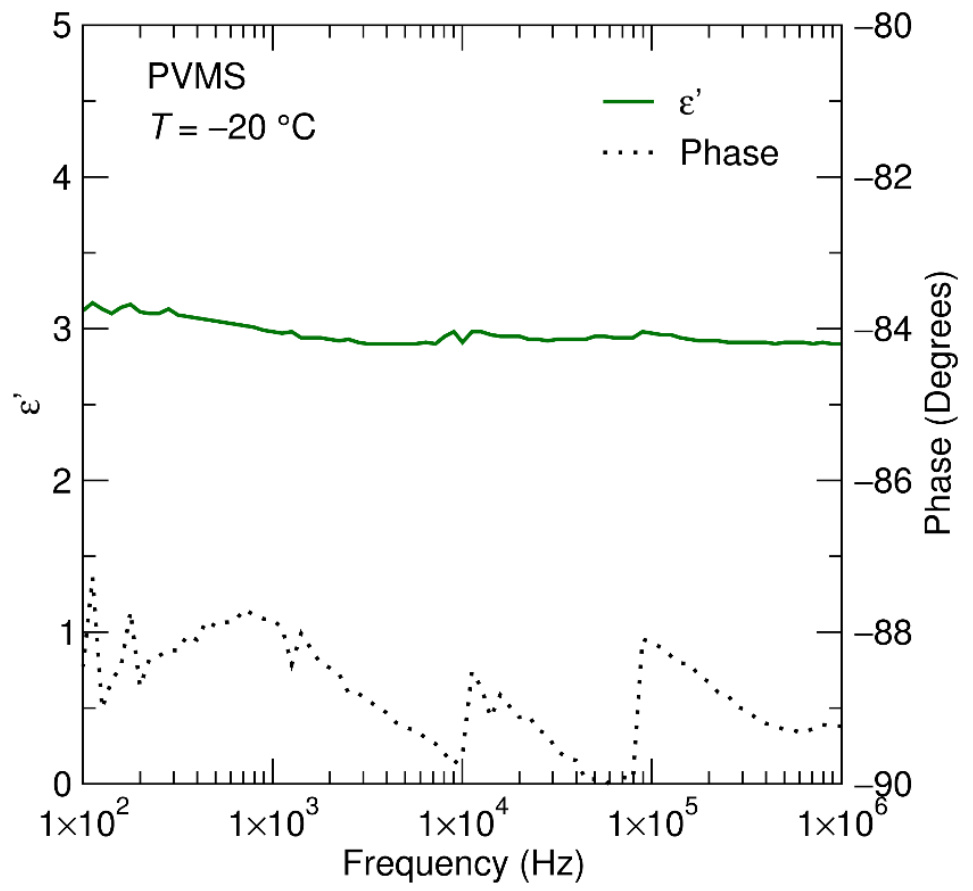

Figure S19. Dielectric constant and phase as a function of frequency for PVMS at $-20{ }^{\circ} \mathrm{C}$. Dielectric constants in Table 1 are reported at $1 \mathrm{MHz}$. 


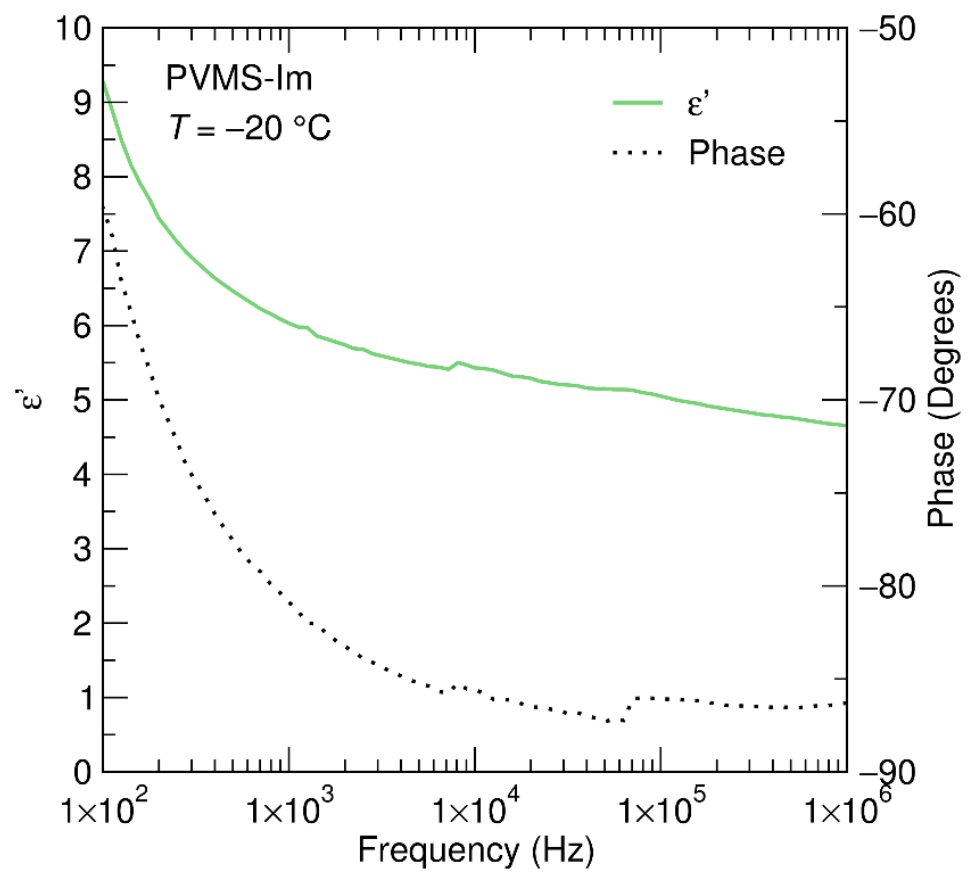

Figure S20. Dielectric constant and phase as a function of frequency for PVMS-Im at $-20{ }^{\circ} \mathrm{C}$. Dielectric constants in Table 1 are reported at $1 \mathrm{MHz}$.

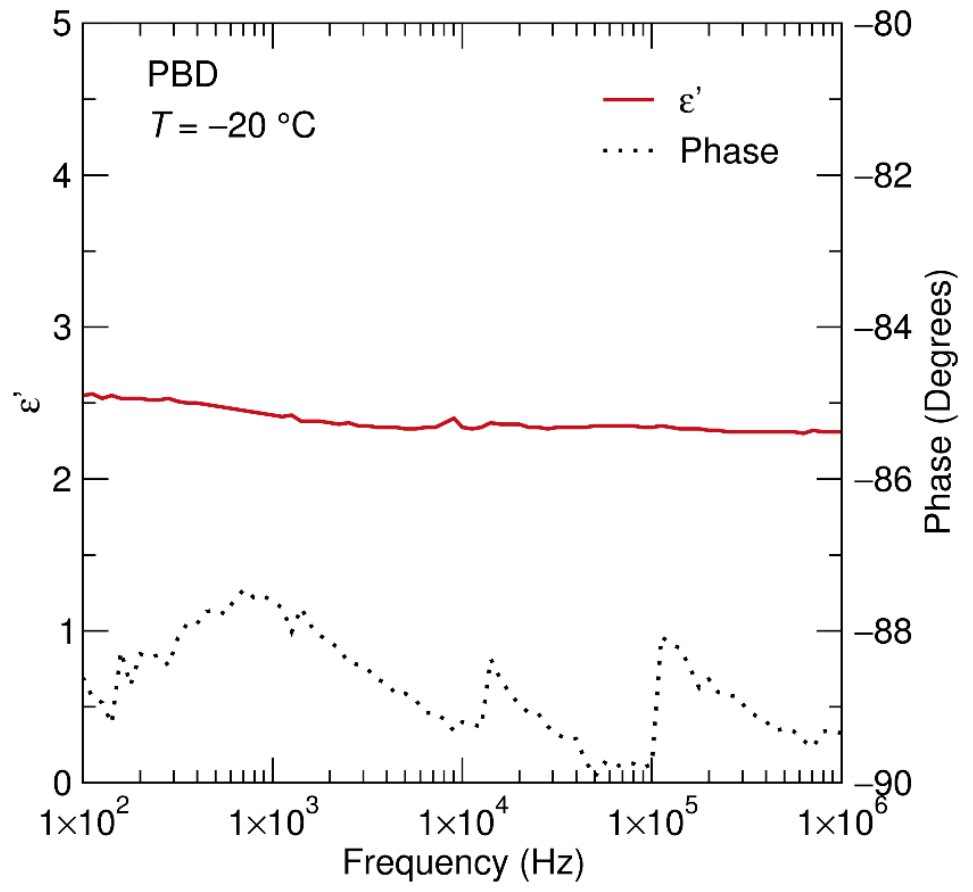

Figure S21. Dielectric constant and phase as a function of frequency for PBD at $-20{ }^{\circ} \mathrm{C}$. Dielectric constants in Table 1 are reported at $1 \mathrm{MHz}$. 


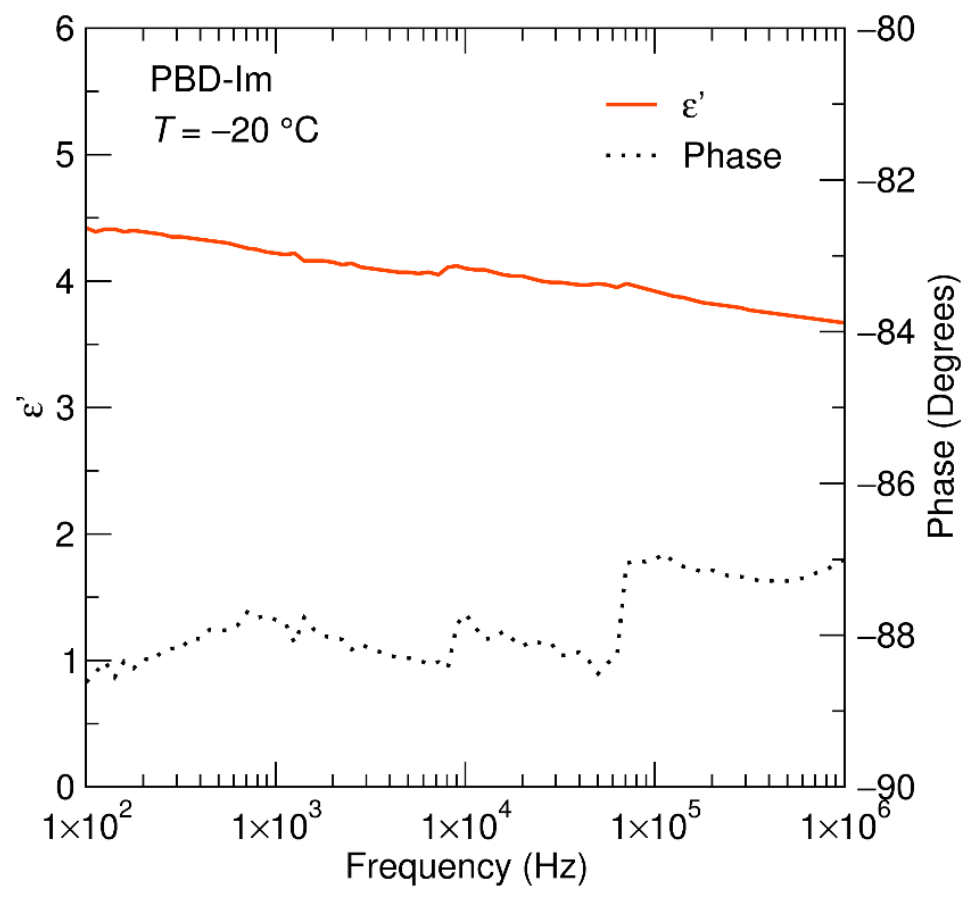

Figure S22. Dielectric constant and phase as a function of frequency for PBD-Im at $-20{ }^{\circ} \mathrm{C}$. Dielectric constants in Table 1 are reported at $1 \mathrm{MHz}$.

\section{X-ray Scattering Characterization}

Separation distance for evenly dispersed ions

For a molar ratio of $\mathrm{Li}^{+}, \mathrm{Cu}^{2+}$, or $\mathrm{Zn}^{2+}$ to imidazole of $r=0.03$, and assuming a polymer density

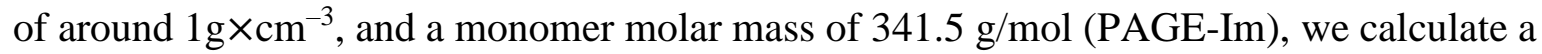
concentration of $\mathrm{M}^{\mathrm{z+}}$ in the polymer as follows:

$$
\begin{gathered}
\frac{1 \mathrm{~g}}{\mathrm{~cm}^{3}} \times \frac{\mathrm{cm}^{3}}{10^{24} \AA^{3}} \times \frac{1 \text { mol polymer }}{341.5 \mathrm{~g}} \times \frac{1 \text { mol imidazole }}{1 \text { mol polymer }} \times \frac{0.03 \text { mol } \mathrm{M}^{z+}}{1 \mathrm{~mol} \text { imidazole }} \times \frac{6.02 \times 10^{23} \text { ions }}{1 \mathrm{~mol} \mathrm{M}^{z+}} \\
=0.00005 \frac{\mathrm{M}^{z+}}{\AA^{3}}=0.05 \frac{\mathrm{M}^{z+}}{\mathrm{nm}^{3}}
\end{gathered}
$$

This corresponds to one metal ion in $20 \mathrm{~nm}^{3}$, or an average separation distance of $20^{1 / 3} \mathrm{~nm}$ or $2.7 \mathrm{~nm}$. A similar calculation can be performed for the other salt concentrations and polymers, with the results listed in Table $S 1$ below. 
Table S1. Approximate cation separation distances expected for evenly dispersed ions for the three polymers and three concentrations studied.

\begin{tabular}{|l|l|l|l|}
\hline Polymer & $r=0.03$ & $r=0.08$ & $r=0.12$ \\
\hline PAGE-Im & $2.7 \mathrm{~nm}$ & $1.9 \mathrm{~nm}$ & $1.7 \mathrm{~nm}$ \\
\hline PVMS-Im & $2.6 \mathrm{~nm}$ & $1.9 \mathrm{~nm}$ & $1.6 \mathrm{~nm}$ \\
\hline PBD-Im & $2.5 \mathrm{~nm}$ & $1.8 \mathrm{~nm}$ & $1.6 \mathrm{~nm}$ \\
\hline
\end{tabular}

Below we show the circular averages for the various polymers, salts and concentrations probed in this study. Figures S26 - S35 show the scattering profiles as a function of temperature for those samples that showed aggregation peaks; for those samples which did not show any aggregation, this did not change with elevated temperature.

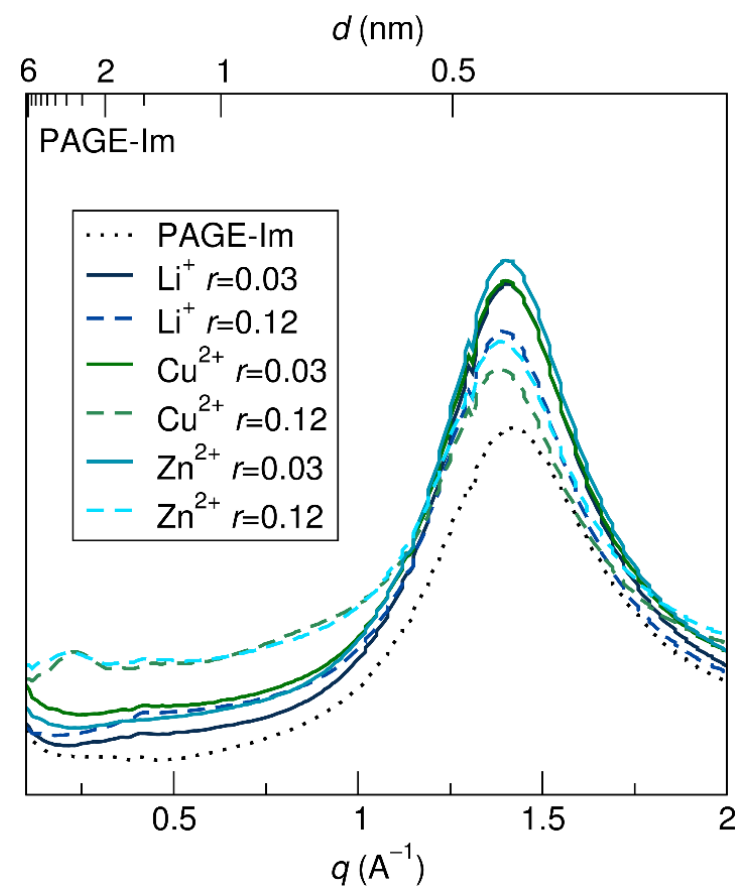

Figure S23. X-ray scattering profile for PAGE-Im at $25^{\circ} \mathrm{C}$ with varying salt identities and concentrations, showing a small aggregation peak around $0.22 \AA^{-1}$ and an amorphous halo around $1.4 \AA^{-1}$. 


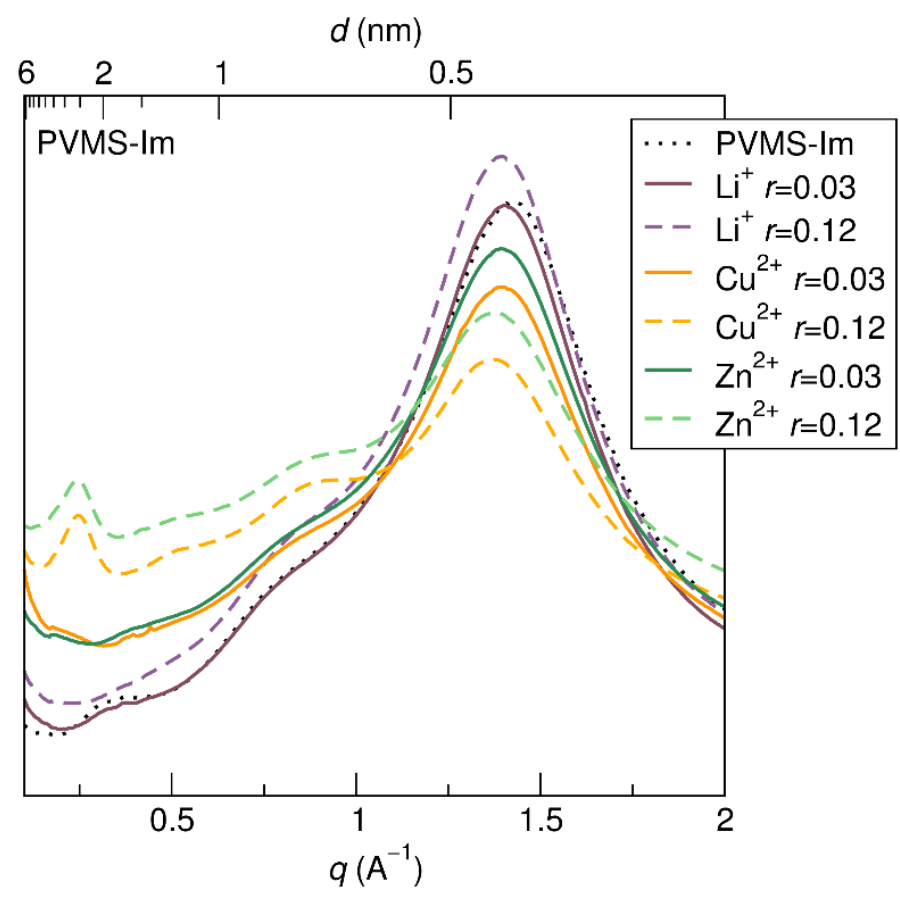

Figure S24. X-ray scattering profile for PVMS-Im at $25^{\circ} \mathrm{C}$ with varying salt identities and concentrations, showing an aggregation peak between 0.15 and $0.25 \AA^{-1}$ and an amorphous halo around $1.4 \AA^{-1}$.

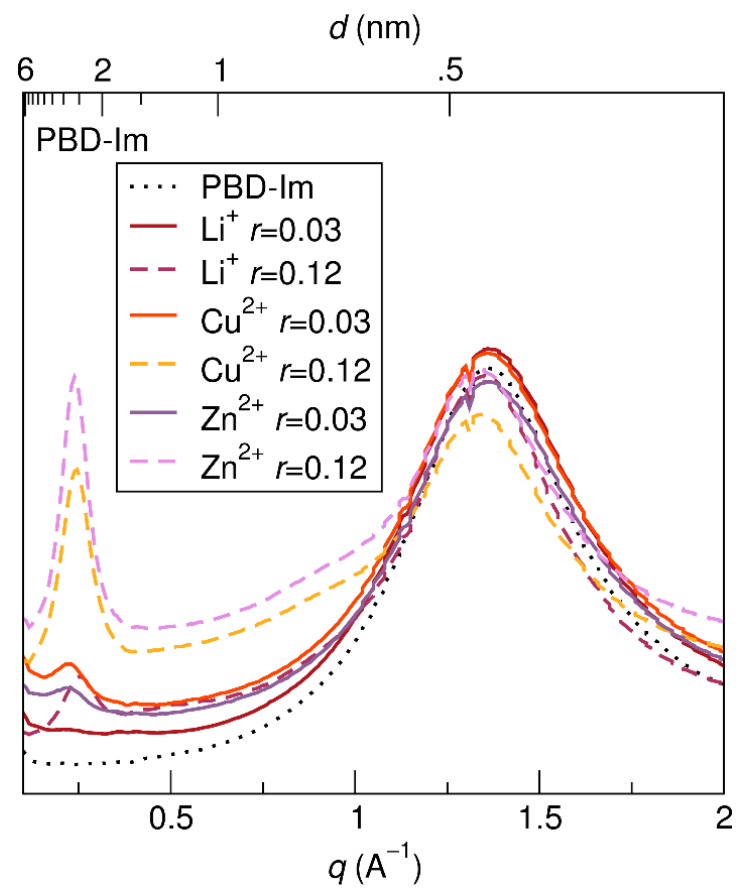

Figure S25. X-ray scattering profile for PBD-Im at $25{ }^{\circ} \mathrm{C}$ with varying salt identities and concentrations, showing an aggregation peak between 0.2 and $0.3 \AA^{-1}$ and an amorphous halo around $1.4 \AA^{-1}$. 


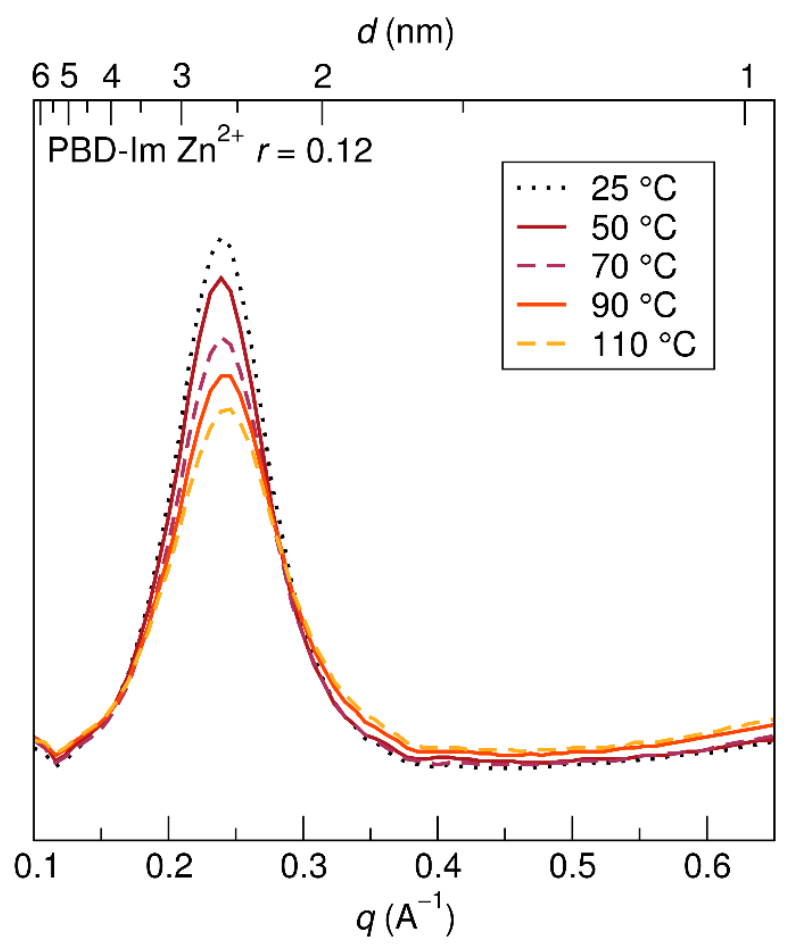

Figure S26. X-ray scattering profile for PBD-Im $\mathrm{Zn}(\mathrm{TFSI})_{2}$ with a molar ratio of $\mathrm{Zn}^{2+}$ :imidazole of 0.12 as a function of temperature shows a reduction in the intensity of the correlation peak centered around $0.24 \AA^{-1}$. This behavior is representative of all samples showing aggregation probed as a function of temperature.

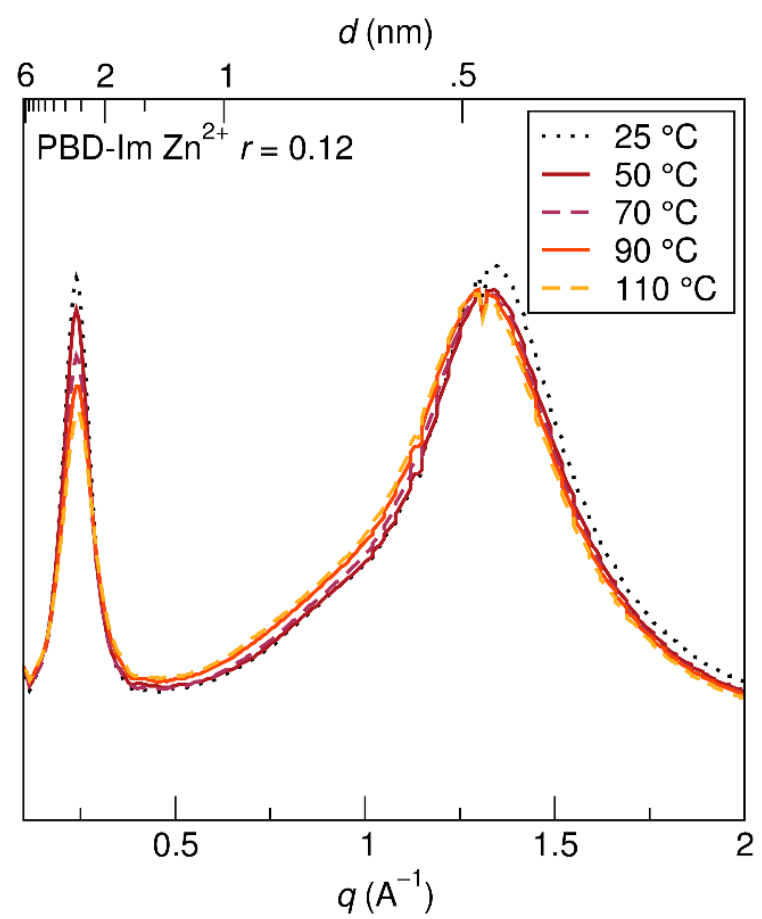

Figure S27. The full X-ray scattering profile for the sample PBD-Im $\mathrm{Zn}^{2+} r=0.12$ sample shown in Figure S26. 


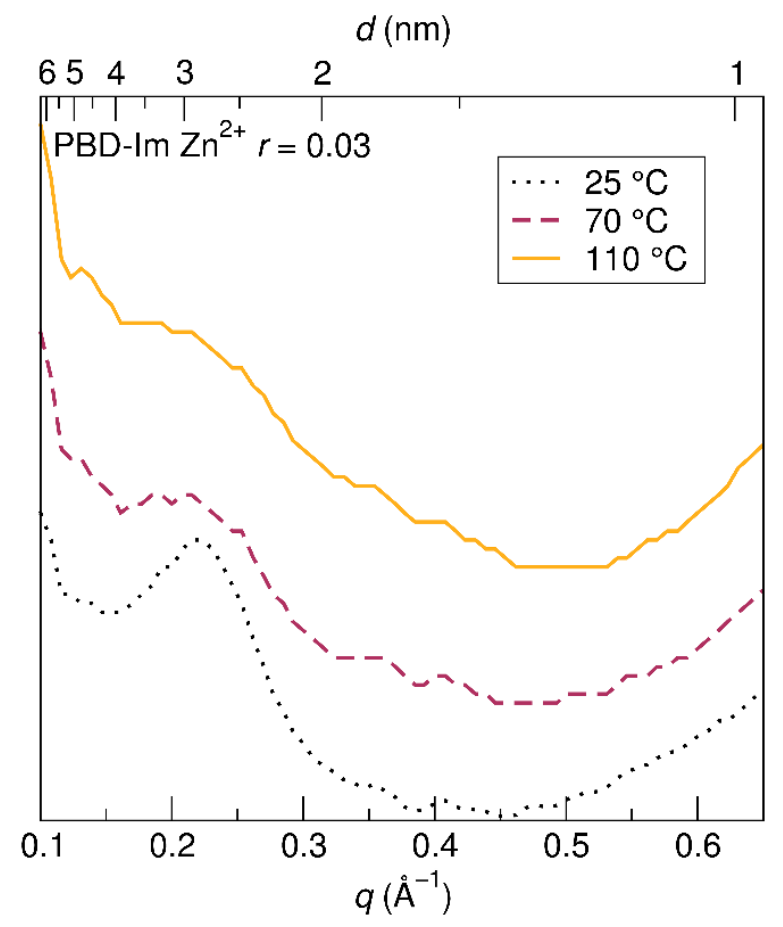

Figure S28. X-ray scattering at $25^{\circ} \mathrm{C}, 70{ }^{\circ} \mathrm{C}$, and $110{ }^{\circ} \mathrm{C}$ for PBD-Im $\mathrm{Zn}^{2+} r=0.03$. The scattering peak diminishes in intensity at elevated temperatures.

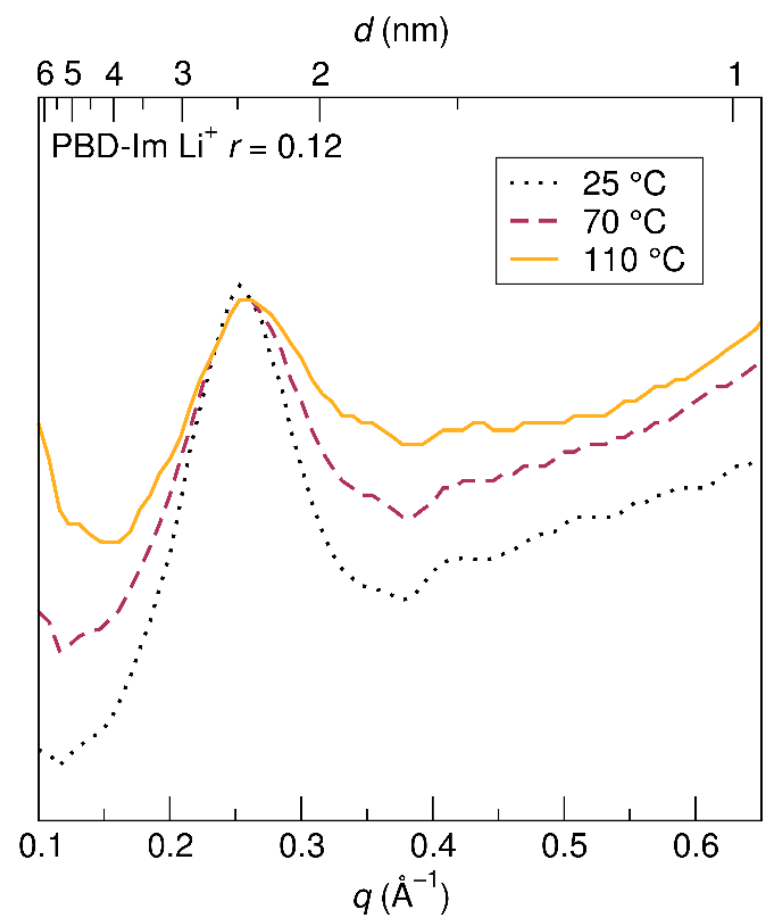

Figure S29. X-ray scattering at $25^{\circ} \mathrm{C}, 70{ }^{\circ} \mathrm{C}$, and $110{ }^{\circ} \mathrm{C}$ for for PBD-Im $\mathrm{Li}^{+} r=0.12$. 


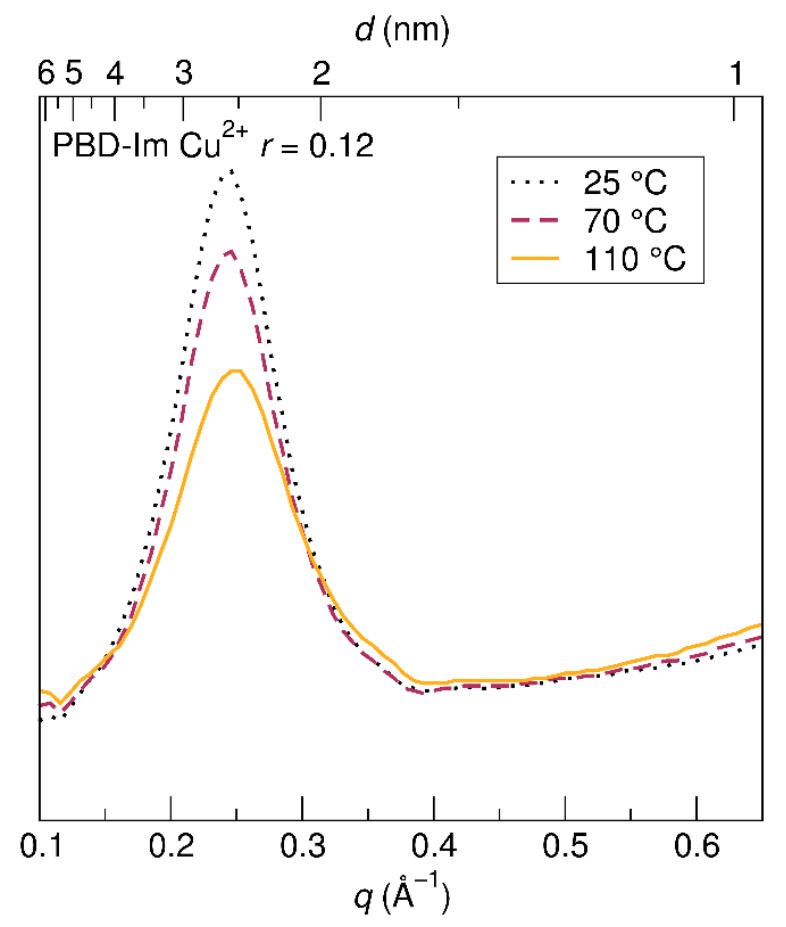

Figure S30. X-ray scattering at $25^{\circ} \mathrm{C}, 70{ }^{\circ} \mathrm{C}$, and $110^{\circ} \mathrm{C}$ for for PBD-Im $\mathrm{Cu}^{2+} r=0.12$.

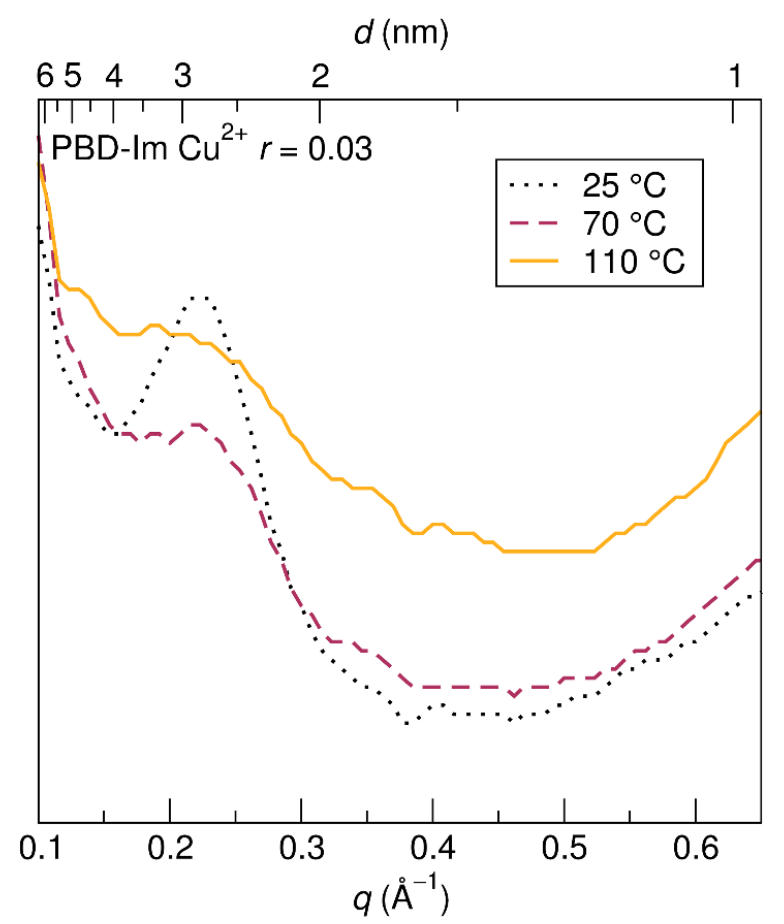

Figure S31. X-ray scattering at $25^{\circ} \mathrm{C}, 70{ }^{\circ} \mathrm{C}$, and $110^{\circ} \mathrm{C}$ for for PBD-Im $\mathrm{Cu}^{2+} r=0.03$. The scattering peak diminishes in intensity at elevated temperatures. 


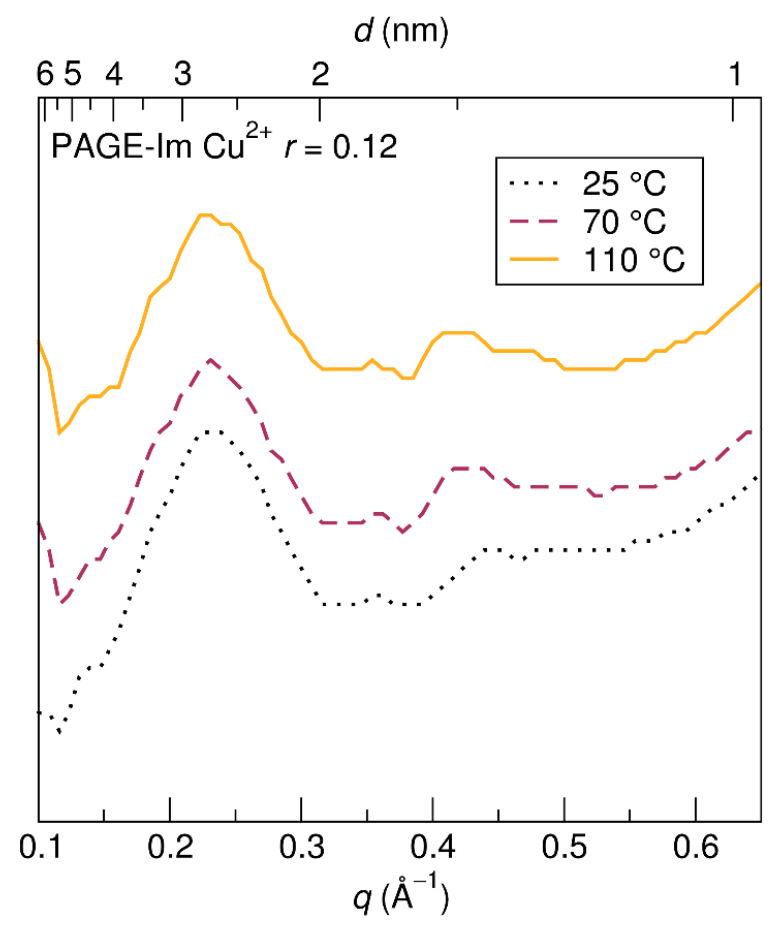

Figure S32. X-ray scattering at $25{ }^{\circ} \mathrm{C}, 70{ }^{\circ} \mathrm{C}$, and $110{ }^{\circ} \mathrm{C}$ for for PAGE-Im $\mathrm{Cu}^{2+} r=0.12$

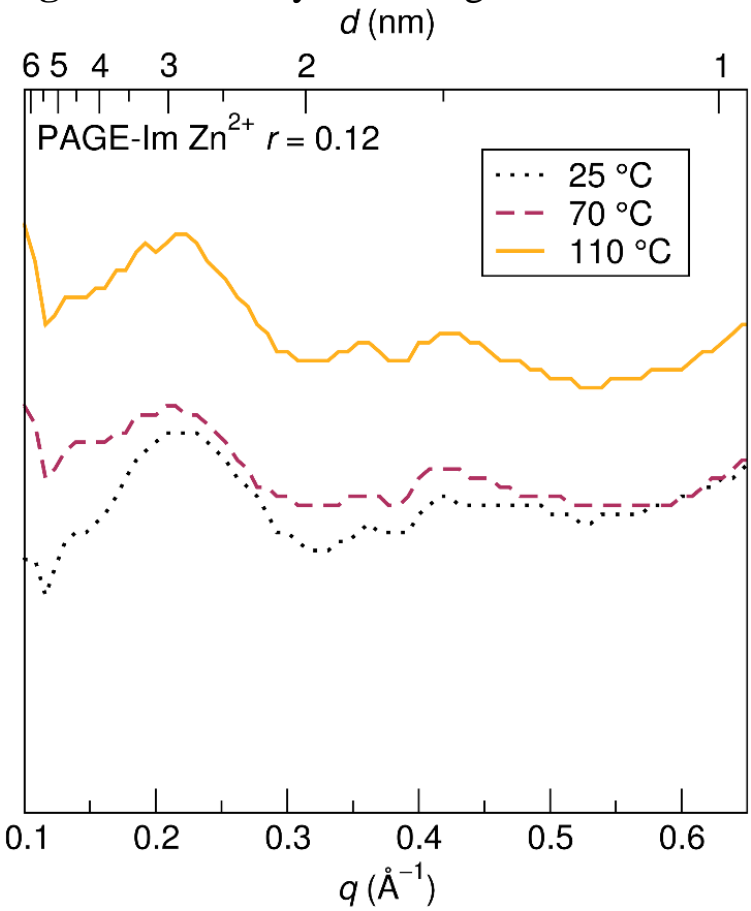

Figure S33. X-ray scattering at $25^{\circ} \mathrm{C}, 70{ }^{\circ} \mathrm{C}$, and $110^{\circ} \mathrm{C}$ for for PAGE-Im $\mathrm{Zn}^{2+} r=0.12$. 


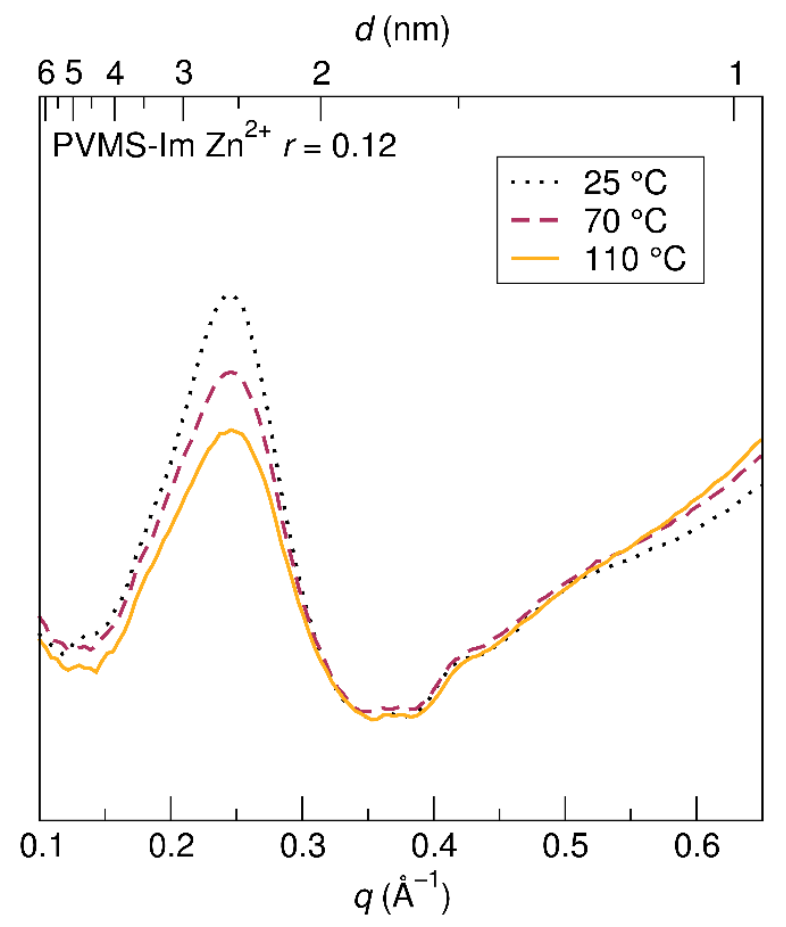

Figure S34. X-ray scattering at $25^{\circ} \mathrm{C}, 70{ }^{\circ} \mathrm{C}$, and $110{ }^{\circ} \mathrm{C}$ for for PVMS-Im $\mathrm{Zn}^{2+} r=0.12$.

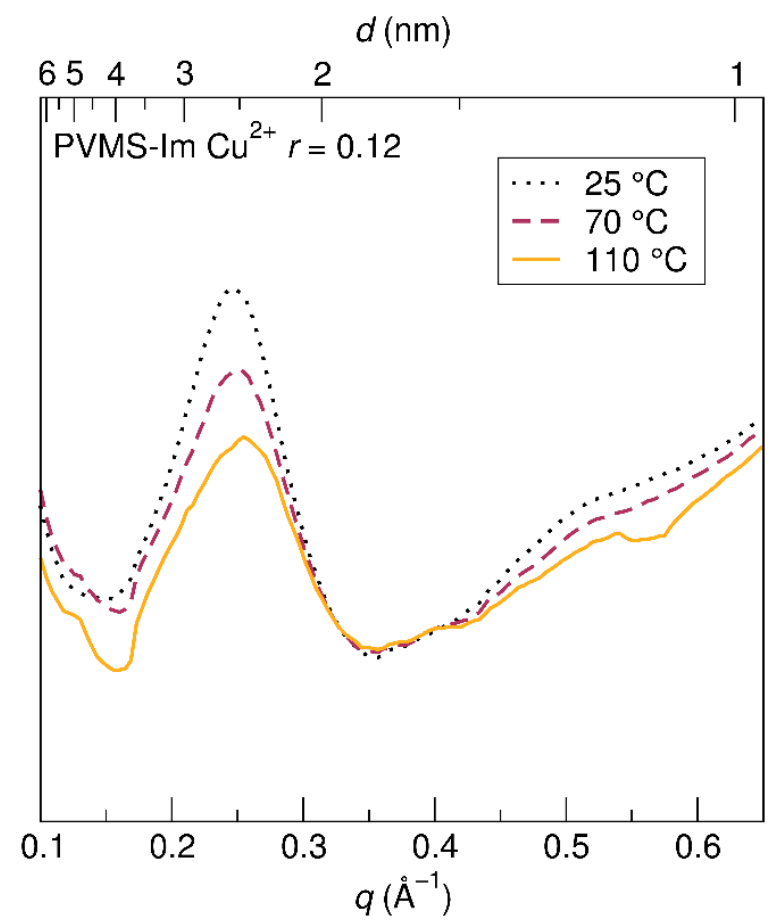

Figure S35. X-ray scattering at $25^{\circ} \mathrm{C}, 70{ }^{\circ} \mathrm{C}$, and $110{ }^{\circ} \mathrm{C}$ for for PVMS-Im $\mathrm{Cu}^{2+} r=0.12$. 


\section{Electron Paramagnetic Resonance}

Field-Swept Echo (FSE)

The FSE spectra for PBD-Im and PAGE-Im were simulated with two components: i) resolved, isolated, $\mathrm{Cu}^{2+}$ ions with an axially elongated tetragonal ligand field $\left(\mathrm{g}_{\|}>\mathrm{g}_{\perp}\right)$ and ii) a very broad, unresolved component.
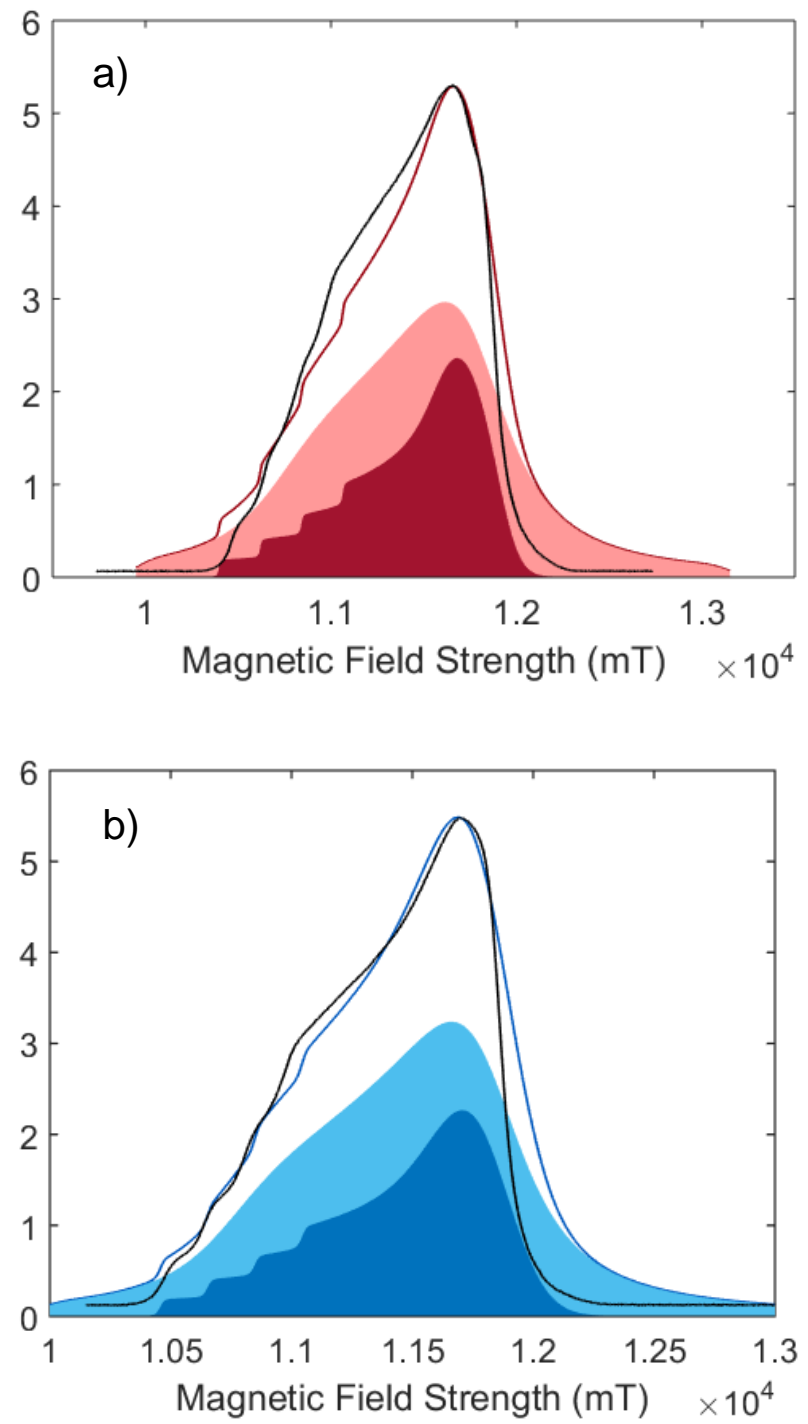

Figure S36. FSE spectra of a) PBD-Im $\mathrm{Cu}^{2+}$ and b) PAGE-Im $\mathrm{Cu}^{2+}$ and the individual spectral contributions from the narrow (isolated) and broad (aggregated) components. 


\section{$\mathrm{Cu}^{2+}$ calculations for dipolar broadening}

A minimum concentration of $\mathrm{Cu}^{2+}$ ions that would cause dipolar broadening even in the absence of aggregation can be calculated assuming random close packing of $\mathrm{Cu}^{2+}$ spheres where the diameter of the $\mathrm{Cu}^{2+}$ sphere is the maximum separation distance that would cause dipolar broadening. Estimating this distance as $d=15 \AA$ provides an easy baseline. Then, the volume of $\mathrm{a} \mathrm{Cu}^{2+}$ sphere is

$$
V_{C u}=4 / 3 \pi(d / 2)^{2}=235.6 \AA^{3}
$$

The packing density for a randomly close packed system of spheres is around $62.5 \%$; thus, the total volume associated with a single $\mathrm{Cu}^{2+}$ ion (occupied volume and free volume) is

$$
V_{\text {total }}=V_{C u} \times \frac{100}{62.5}=377 \AA^{3}
$$

Thus, there must be at least one $\mathrm{Cu}^{2+}$ in a volume of $377 \AA^{3}$ to observe dipolar broadening without ion aggregation. For a molar ratio of $\mathrm{Cu}^{2+}$ to imidazole of $r=0.03$, and assuming a polymer density of around $1 \mathrm{~g} \times \mathrm{cm}^{-3}$, and a monomer molar mass of $341.5 \mathrm{~g} / \mathrm{mol}$ (PAGE-Im), we calculate a concentration of $\mathrm{Cu}^{2+}$ in the polymer as follows:

$$
\begin{gathered}
\frac{1 \mathrm{~g}}{\mathrm{~cm}^{3}} \times \frac{\mathrm{cm}^{3}}{10^{24} \AA^{3}} \times \frac{1 \text { mol polymer }}{341.5 \mathrm{~g}} \times \frac{1 \text { mol imidazole }}{1 \text { mol polymer }} \times \frac{0.03 \mathrm{~mol} \mathrm{Cu}^{2+}}{1 \text { mol imidazole }} \times \frac{6.02 \times 10^{23} \text { ions }}{1 \text { mol Cu }} \\
=0.00005 \frac{\mathrm{Cu}^{2+}}{\AA^{3}}=0.02 \frac{\mathrm{Cu}^{2+}}{377 \AA^{3}}
\end{gathered}
$$

Thus, there is a much lower $\mathrm{Cu}^{2+}$ concentration in the polymer than what would be required to reach dipolar broadening without ion aggregation.

\section{Hyperfine Sublevel Correlation Spectroscopy (HYSCORE)}

Two-dimensional hyperfine sublevel correlation (HYSCORE) spectroscopy is a well-established technique that provides correlations between nuclear frequencies of different spin manifolds that interact with the unpaired electron. ${ }^{1}$ In other words, it shows the NMR transitions of magnetically active nuclei interacting with the electron spin. The ${ }^{14} \mathrm{~N}$ nucleus is magnetically active, having a nuclear spin $I=1$, in principle should result in six nuclear transitions if it interacts with an unpaired electron, three from each electron spin manifold, $m_{\mathrm{s}}= \pm 1 / 2$. For a disordered system such as PAGEIm and PBD-Im, we expect complicated spectra, with large anisotropy leading to broadened peaks on the HYSCORE spectrum. These peaks contain information about the strength and nature of the hyperfine interaction between the unpaired spin in the $\mathrm{Cu}^{2+}$ nucleus and ${ }^{14} \mathrm{~N}$. The hyperfine interaction consists of two components, $\mathrm{a}_{\text {iso }}$ and $T$. $\mathrm{a}_{\text {iso, }}$, isotropic hyperfine coupling, is the Fermi contact term, which measures the spin density on the nucleus; while $\mathrm{T}$, the dipolar coupling term, is inversely proportional to the distance between the electron and the nuclear spins $\left(\mathrm{T} \alpha 1 / \mathrm{r}^{3}\right)$. In addition, since ${ }^{14} \mathrm{~N}$ is a spin $>1 / 2(\mathrm{I}=1)$ nucleus, it has a quadrupole moment that interacts with the electric field gradient (EFG) around the nucleus. The EFG tensor consists of 3 components. This 
EFG tensor can be fully described using two parameters, the nuclear quadrupole coupling constant, $K=e^{2} q_{z z} Q / h$, where $e$ is the charge of the electron and $Q$ is the nuclear electric quadrupole moment of the ${ }^{14} \mathrm{~N}$ nucleus, and the asymmetry parameter, $\eta$. $K$ measures the strength of interaction between the nuclear quadrupole moment and the EFG at the nucleus due to anisotropic distribution of charges. On the other hand, $\eta$ measures the deviation of the distribution of charges from axial symmetry. $K$ and $\eta$, along with $a_{\text {iso, }}$ are used as signatures to identify the chemical environment around the ${ }^{14} \mathrm{~N}$ nucleus, allowing its characterization. Extensive studies on $\mathrm{Cu}^{2+}$ and its interactions with the remote ${ }^{14} \mathrm{~N}$ of the imidazole have allowed the elucidation of characteristic parameters for these systems. $^{2-6}$ Experiments at X-band $(9.5 \mathrm{GHz})$ i.e. lower fields, typically fulfill the cancellation condition, where the three nuclear frequencies of each electron spin manifold, equal the three pure nuclear quadrupole resonance frequencies. In addition, a broadened double quantum transition, $v_{\mathrm{dq}}$ is also observed. This frequency is correlated with $K$, $\mathrm{a}_{\text {iso }}$ and $\eta$ using the following equation:

$$
v_{\mathrm{dq} \pm}=2 \sqrt{\left(v_{\mathrm{I}} \pm \frac{\mathrm{a}_{\mathrm{iso}}}{2}\right)^{2}+\left(\frac{K}{4}\right)^{2}\left(3+\eta^{2}\right)}
$$

Therefore, HYSCORE experiments that meet the cancellation conditions exhibit cross-peaks that correlate the three nuclear quadrupole resonances with $v_{\mathrm{dq}}$. Under X-Band conditions, this results in intrinsically complex spectra. When the cancellation condition not fulfilled, for example, at QBand frequencies $(35 \mathrm{GHz})$, the HYSCORE spectra are greatly simplified and only exhibit a single pair of cross-peaks of the $v_{\mathrm{dq}}$ transitions. ${ }^{7}$ To simplify spectral analysis for such disordered systems, HYSCORE experiments were done at Q-Band frequencies.

As discussed previously, pulsed EPR allows the investigation of couplings at specific magnetic fields which correspond to different orientations of the electron spins in the polymers. The maximum peak occurs at a magnetic field (observer position) $\mathrm{B}_{0}$ of $11650 \mathrm{G}$, which corresponds to a maximum population of spins at that particular orientation $\left(\mathrm{g}_{\perp}\right)$. Experimental spectra were also recorded at other observer field positions and decent fits were obtained for each of the polymers (Figure S37-38). The presence of characteristic dq cross-peaks at approximately [5.05, 9.10] $\mathrm{MHz}$ and $[9.10,5.05] \mathrm{MHz}$ in the strong coupling regime $\left(\mathrm{A}>\mathrm{v}_{14 \mathrm{~N}} / 2\right)$ shows direct coordination of ${ }^{14} \mathrm{~N}$ species to the unpaired electrons in $\mathrm{Cu}^{2+}$ in both polymer backbones. In addition, a broad anisotropic peak appears in the lower frequency regime centered around [3.6, 3.6] $\mathrm{MHz}$ for PAGE-Im $\mathrm{Cu}^{2+} . \mathrm{Cu}^{2+}$-imidazole systems typically show a iso constants of $1.5-2 \mathrm{MHz}$, $\mathrm{K}$ values between $1.4-2 \mathrm{MHz}$, and $\eta$ parameters of $0.8-1$, which were used as starting points for the simulated spectra. ${ }^{2-6}$

The experimental parameters, such as magnetic field, excitation frequency, number of points, dwell time, pulse width, and pulse delay, were taken directly from the HYSCORE experiments. Simulations of the spectra were iteratively done until a set of parameters was obtained that could reproduce the general features of the experimental spectra. The fit was performed using one type of ${ }^{14} \mathrm{~N}$ species, where each of the five parameters $\left(\mathrm{a}_{\mathrm{iso}}, \mathrm{T}, \mathrm{K}, \eta\right.$ and $\beta$ ) were varied until a reasonable fit was observed for PBD-Im $\mathrm{Cu}^{2+}$ and fell within expected values for imidazole ligands (Table $\mathrm{S} 2$ ). The estimated $\mathrm{a}_{\text {iso }}$ value of $2 \mathrm{MHz}$ corresponds to an unpaired spin density in the ${ }^{14} \mathrm{~N} 2 \mathrm{~s}$ orbital of $\sim 1 \times 10^{-3}$ arising from the coordination of the imidazole ligand. The dipolar coupling term is not dominant for imidazole ${ }^{14} \mathrm{~N}(0.15 \mathrm{MHz})$, while the $\mathrm{K}$ and $\eta$ terms equal $1.8 \mathrm{MHz}$ and 0.8 
respectively. On the other hand, spectra for PAGE-Im $\mathrm{Cu}^{2+}$ could not be reproduced with the same parameters, especially at the low frequency region. Another ${ }^{14} \mathrm{~N}$ component was therefore introduced to obtain a reasonable fit between the experimental and simulated spectra. The estimated parameters for this ${ }^{14} \mathrm{~N}$ component also fall within the range of values that correspond to imidazole ligands, albeit with smaller $\mathrm{a}_{\text {iso }}(1.5 \mathrm{MHz})$ and $\mathrm{K}(1.5 \mathrm{MHz})$ values. The $\mathrm{a}_{\text {iso }}$ value describes the delocalization of electrons in the imidazole ring, therefore hinting towards slightly less delocalization for one of the imidazole environments. In addition, the $\mathrm{K}$ value is also smaller, suggesting a more symmetric environment. Finally, the orientation of this second imidazole, obtained from the Euler angle of the nuclear quadrupole tensor is almost perpendicular to that of the first. The principal axes system (PAS) specific to a molecule is used as the molecular frame reference. The PAS for $\mathrm{Cu}$-imidazole complexes is typically the g parallel axis/symmetry axis. An estimation of the orientation of the hyperfine and the quadrupole coupling tensor (from the ${ }^{14} \mathrm{~N}$ of the imidazole) can be found from the Euler angle $(\alpha, \beta, \gamma)$ because that angle can be thought of as a rotation angle to the molecular frame reference. In these simulations, the hyperfine angles did not significantly change the spectrum. However, varying the $\beta$ angle of the quadrupole tensor played a role in the appearance of the peaks.
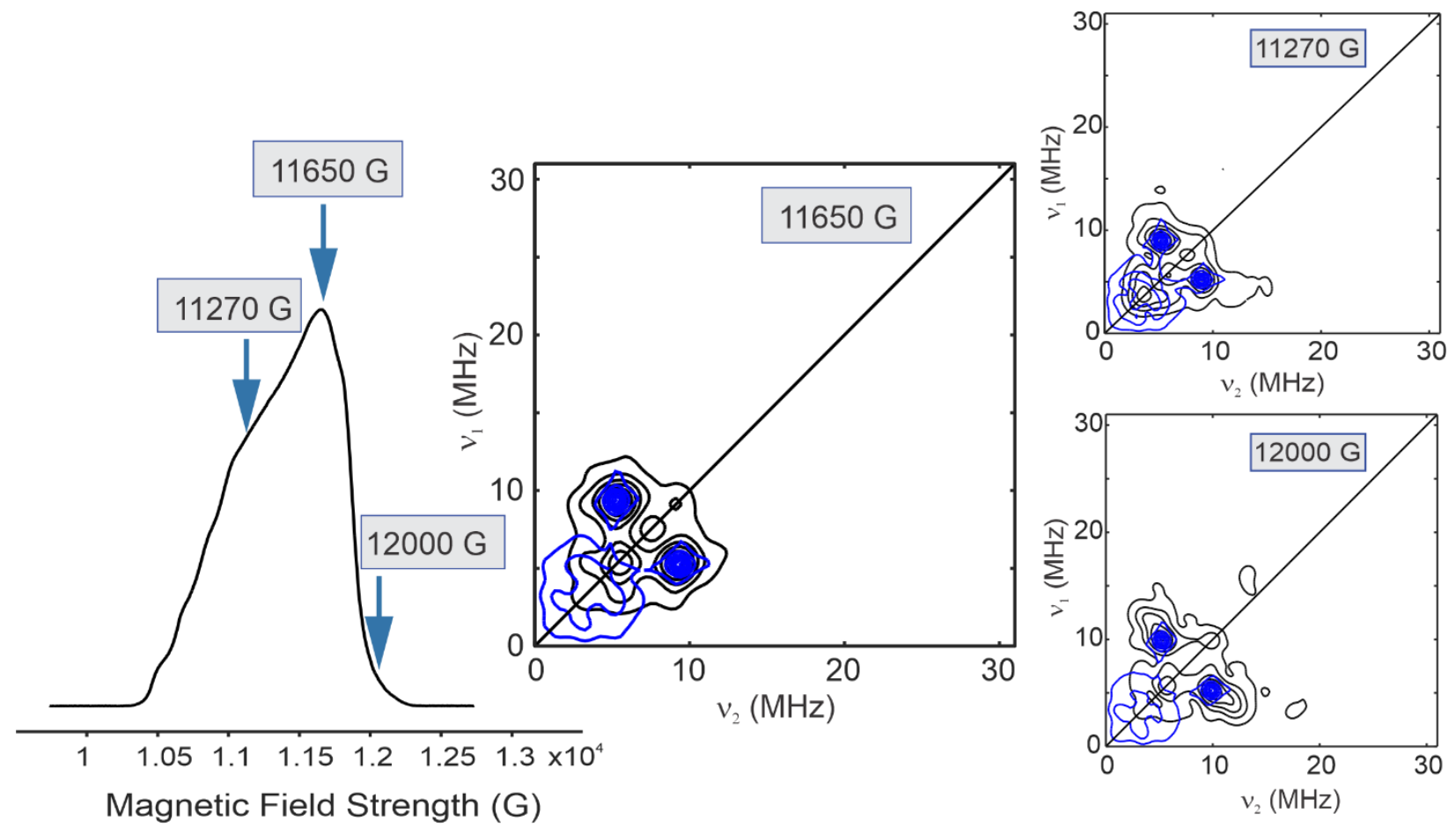

Figure S37. HYSCORE spectra of PBD-Im $\mathrm{Cu}^{2+}$ collected at different observer field positions and their corresponding simulations using the same set of parameters. 

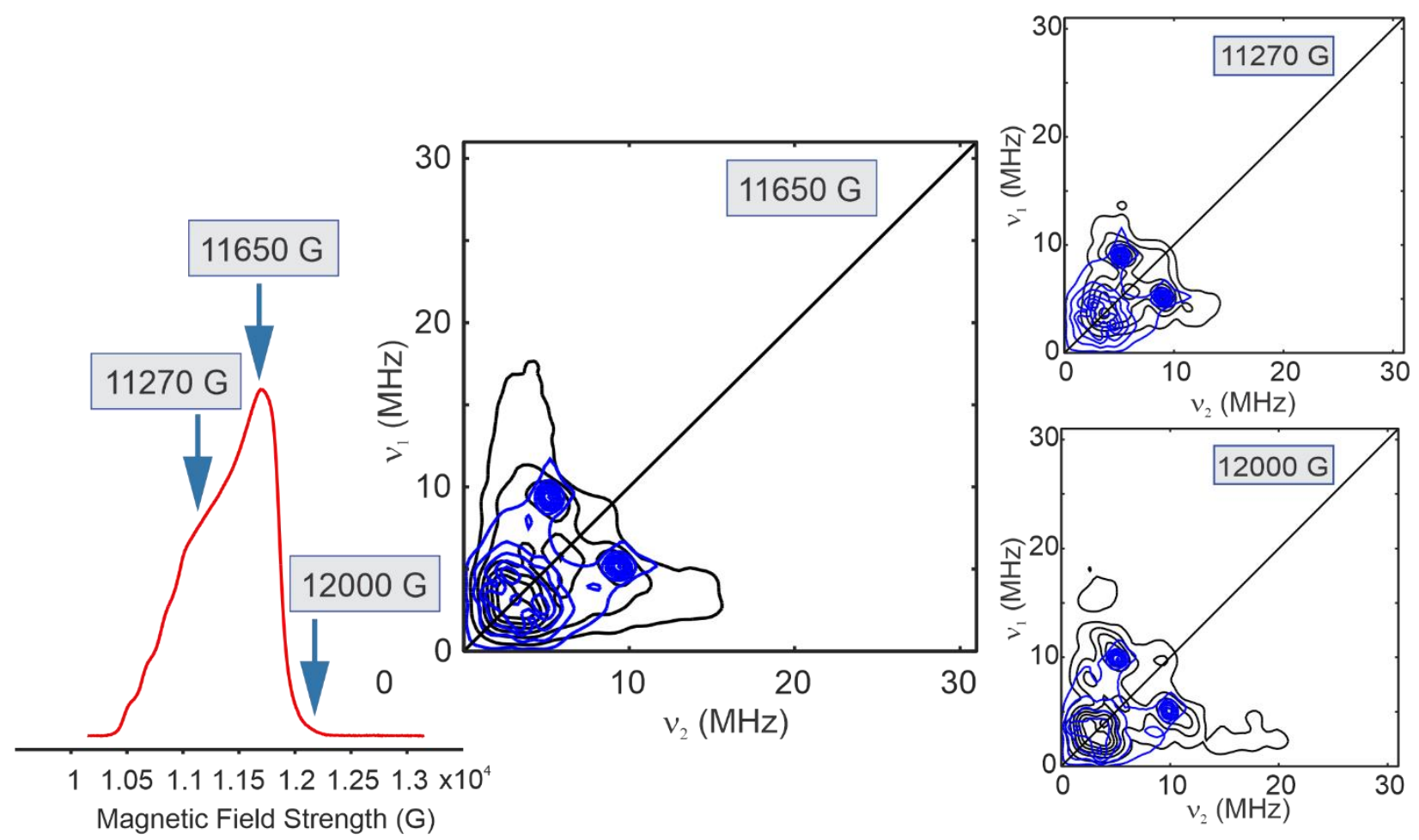

Figure S38. HYSCORE spectra of PAGE-Im $\mathrm{Cu}^{2+}$ collected at different observer field positions and their corresponding simulations using the same set of parameters.

Table S2. Estimated ${ }^{14} \mathrm{~N}$ HYSCORE parameters from simulations of experimental data.

\begin{tabular}{|c|c|c|c|c|c|}
\hline & $\mathrm{a}_{\text {iso }}(\mathrm{MHz})$ & $\mathrm{T}(\mathrm{MHz})$ & $\mathrm{K}$ & $\eta$ & $\mathrm{B}\left(^{\circ}\right)$ \\
\hline PBD-Im $\mathrm{Cu}^{2+}$ & 2 & 0.15 & 1.8 & 0.8 & 80 \\
\hline PAGE-Im $\mathrm{Cu}^{2+}(\mathrm{I})$ & 1.5 & 0.2 & 1.5 & 0.8 & 0 \\
\hline PAGE-Im $\mathrm{Cu}^{2+}(\mathrm{II})$ & 2 & 0.15 & 1.8 & 0.8 & 80 \\
\hline
\end{tabular}




\section{Field-theoretic (complex Langevin) simulations}

Using Hubbard-Stratonovich transformations, the field-theoretic canonical partition function for the system of metal salt-coordinating polymers takes the form ${ }^{8}$

$$
Z=Z_{0} \int \mathcal{D} w_{1} \int \mathcal{D} w_{2} \int \mathcal{D} w_{3} \int \mathcal{D} w_{4} \int \mathcal{D} \varphi e^{-H\left[w_{1}, w_{2}, w_{3}, w_{4}, \varphi\right]},
$$

where $\varphi$ is an electrostatic field that mediates the ion-ion, ion-dipole and dipole-dipole interactions, and $w_{1}, w_{2}, w_{3}$, and $w_{4}$ are exchange-mapped chemical potential fields that mediate contact interactions in the model. These contact interactions include the excluded volume interactions, as well as the Flory interactions between species $\mathrm{A}$ and $\mathrm{B}\left(\chi_{A B}=0.2\right)$, between $\mathrm{B}$ and the cation $\left(\chi_{B+}=-20\right)$, and between the cation and anion $\left(\chi_{+-}=0.2\right)$. The parameter $\chi_{+-}$ does not have a significant effect on the structure but helps to reduce numerical scatter in the ionion structure factors that we measure. The term $Z_{0}$ contains ideal gas terms, normalization factors from the Hubbard-Stratonovich transforms, and self-interaction corrections, and its precise form is of no consequence here. The Hamiltonian $H\left[w_{1}, w_{2}, w_{3}, w_{4}, \varphi\right]$ is given by

$$
\begin{aligned}
& H\left[w_{1}, w_{2}, w_{3}, w_{4}, \varphi\right] \\
& \quad=\frac{\rho_{0}}{2} \sum_{i=1}^{4} \frac{1}{\sigma_{i}} \int d \boldsymbol{r} w_{i}^{2}(\boldsymbol{r})+\frac{1}{8 \pi l_{B}^{(0)}} \int d \boldsymbol{r}|\nabla \varphi|^{2}-n_{p} \ln Q_{p}\left[\Omega_{A}, \Omega_{B}\right]-n_{+} \ln Q_{+}\left[\Omega_{+}\right] \\
& -n_{-} \ln Q_{-}\left[\Omega_{-}\right],
\end{aligned}
$$

where $Q_{l}$ is the single-molecule partition function for molecular species $l$ and $\Omega_{m}$ is the local chemical potential field experienced by beads of species $m$. In general, the $\Omega_{m}$ contain contributions from all five auxiliary fields, and are given by

$$
\begin{gathered}
\Omega_{A}(\boldsymbol{r})=\sum_{i=1}^{4} \xi_{i} \Phi_{i A} \bar{w}_{i}(\boldsymbol{r})+\frac{\alpha_{A}}{2 \beta e^{2}}|\nabla \bar{\varphi}|^{2}, \\
\Omega_{B}(\boldsymbol{r})=\sum_{i=1}^{4} \xi_{i} \Phi_{i B} \bar{w}_{i}(\boldsymbol{r})+\frac{\alpha_{B}}{2 \beta e^{2}}|\nabla \bar{\varphi}|^{2}, \\
\Omega_{ \pm}(\boldsymbol{r})=\sum_{i=1}^{4} \xi_{i} \Phi_{i \pm} \bar{w}_{i}(\boldsymbol{r}) \mp i z \bar{\varphi}(\boldsymbol{r}) .
\end{gathered}
$$

Here $\sigma_{i}$ and $\Phi_{i j}$ are the eigenvalues and eigenvectors of the contact interaction matrix, respectively, and the parameter $\xi_{i}$ takes the value 1 if $\sigma_{i}<0$ and the value $i$ if $\sigma_{i}>0$. The overbar on the fields indicates a spatial convolution with the Gaussian smearing function, for example:

$$
\bar{\varphi}(\boldsymbol{r})=\int d \boldsymbol{r}^{\prime} \Gamma\left(\boldsymbol{r}-\boldsymbol{r}^{\prime}\right) \varphi\left(\boldsymbol{r}^{\prime}\right)
$$

where $\Gamma(\boldsymbol{r})=\left(2 \pi a^{2}\right)^{-3 / 2} e^{-r^{2} / 2 a^{2}}$. Since the metal salt-coordinating polymer is architecturally a bottlebrush polymer, the single-chain partition function takes a form similar to that described in several recent works; ${ }^{9-10}$ that is, 


$$
Q_{p}\left[\Omega_{A}, \Omega_{B}\right]=\frac{1}{V} \int d \boldsymbol{r} q_{b b}\left(\boldsymbol{r}, N_{b b}\right),
$$

where $q_{b b}(\boldsymbol{r}, s)$ is the forward backbone propagator. In general, in order to sample the trajectories of the fields via the complex Langevin scheme, and also to calculate the polymer densities, we must compute the forward and backward backbone propagators $q_{b b}(\boldsymbol{r}, s)$ and $\tilde{q}_{b b}(\boldsymbol{r}, s)$, respectively, as well as the forward and backward side-chain propagators $q_{s c}(\boldsymbol{r}, s)$ and $\tilde{q}_{s c}(\boldsymbol{r}, s)$, respectively. If there are $N_{b b} / \tau-1$ equally-spaced side-chains (i.e. a junction every $\Delta s=\tau$ along the backbone), the following modified diffusion equation must be solved in sections of length $\tau$ for the forward backbone propagator $q_{b b}(\boldsymbol{r}, s)=q_{b b}^{(j)}(\boldsymbol{r}, s)$, where $j \tau \leq s<(j+1) \tau$ :

$$
\frac{\partial}{\partial s} q_{b b}^{(j)}(\boldsymbol{r}, s)=\left[\frac{b^{2}}{6} \nabla^{2}-\Omega_{A}(\boldsymbol{r})\right] q_{b b}^{(j)}(\boldsymbol{r}, s)
$$

with a $j$-dependent initial condition given by

$$
q_{b b}^{(j)}(\boldsymbol{r}, j \tau)=\left\{\begin{array}{rr}
1, & j=0 \\
q_{b b}^{(j-1)}(\boldsymbol{r}, j \tau) q_{s c}\left(\boldsymbol{r}, N_{s c}\right), & j \neq 0
\end{array}\right.
$$

and a similar equation for the backward backbone propagator:

$$
\frac{\partial}{\partial s} \tilde{q}_{b b}^{(j)}\left(\boldsymbol{r}, N_{b b}-s\right)=\left[\frac{b^{2}}{6} \nabla^{2}-\Omega_{A}(\boldsymbol{r})\right] \tilde{q}_{b b}^{(j)}\left(\boldsymbol{r}, N_{b b}-s\right),
$$

with the initial condition

$$
\tilde{q}_{b b}^{(j)}\left(\boldsymbol{r}, N_{b b}-j \tau\right)=\left\{\begin{array}{rr}
1, & j=0 \\
\tilde{q}_{b b}^{(j-1)}\left(\boldsymbol{r}, N_{b b}-j \tau\right) q_{s c}\left(\boldsymbol{r}, N_{s c}\right), & j \neq 0
\end{array}\right.
$$

and we also note that if the polymer is symmetric along the backbone contour, as it is in our case, then $q_{b b}(\boldsymbol{r}, s)=\tilde{q}_{b b}\left(\boldsymbol{r}, N_{b b}-s\right)$ by symmetry and the backward backbone propagator need not be computed. The forward side-chain propagator, which we define to propagate starting from the side-chain end, satisfies

$$
\frac{\partial}{\partial s} q_{s c}(\boldsymbol{r}, s)=\left[\frac{b^{2}}{6} \nabla^{2}-\Omega_{m}(\boldsymbol{r})\right] q_{s c}(\boldsymbol{r}, s),
$$

where

$$
\Omega_{m}(\boldsymbol{r})= \begin{cases}\Omega_{B}(\boldsymbol{r}), & s<N_{s c} / 2 \\ \Omega_{A}(\boldsymbol{r}), & s \geq N_{s c} / 2\end{cases}
$$

and with the initial condition $q_{s c}(\boldsymbol{r}, 0)=1$. The backward propagators for the side-chains $\tilde{q}_{s c}^{(j)}(\boldsymbol{r}, s)$, where $j=1,2, \ldots, N_{b b} / \tau-1$, acquire a dependence on the position of their junction along the backbone through their initial condition, and satisfy

$$
\frac{\partial}{\partial s} \tilde{q}_{s c}^{(j)}\left(\boldsymbol{r}, N_{s c}-s\right)=\left[\frac{b^{2}}{6} \nabla^{2}-\Omega_{m}(\boldsymbol{r})\right] \tilde{q}_{s c}^{(j)}\left(\boldsymbol{r}, N_{s c}-s\right),
$$


where

$$
\Omega_{m}(\boldsymbol{r})= \begin{cases}\Omega_{A}(\boldsymbol{r}), & s<N_{s c} / 2 \\ \Omega_{B}(\boldsymbol{r}), & s \geq N_{s c} / 2\end{cases}
$$

and with the initial condition $\tilde{q}_{s c}^{(j)}\left(\boldsymbol{r}, N_{s c}\right)=q_{b b}(\boldsymbol{r}, j \tau) \tilde{q}_{b b}\left(r, N_{b b}-j \tau\right)$. The single-molecule partition functions for the cation and anion take a simpler form, given by

$$
Q_{ \pm}\left[\Omega_{ \pm}\right]=\frac{1}{V} \int d \boldsymbol{r} e^{-\Omega_{ \pm}(r)}
$$

In order to sample this unapproximated field theory we employ complex Langevin sampling, in which the auxiliary fields are promoted to be complex variables that evolve according to the following equations of motion:

$$
\begin{aligned}
\frac{\partial w_{i}(\boldsymbol{r}, t)}{\partial t} & =-\Lambda_{w_{i}} \frac{\delta H[\{w\}, \varphi]}{\delta w_{i}(\boldsymbol{r}, t)}+\eta_{w_{i}}(\boldsymbol{r}, t), \\
\frac{\partial \varphi(\boldsymbol{r}, t)}{\partial t} & =-\Lambda_{\varphi} \frac{\delta H[\{w\}, \varphi]}{\delta \varphi(\boldsymbol{r}, t)}+\eta_{\varphi}(\boldsymbol{r}, t),
\end{aligned}
$$

where the $\eta_{j}(\boldsymbol{r}, t)$ are real-valued Gaussian-distributed white noise variables $(j$ runs over the set of auxiliary fields) with zero mean $\left\langle\eta_{j}(\boldsymbol{r}, t)\right\rangle=0$ and satisfying the fluctuation-dissipation theorem $\left\langle\eta_{i}(\boldsymbol{r}, t) \eta_{j}\left(\boldsymbol{r}^{\prime}, t^{\prime}\right)\right\rangle=2 \Lambda_{j} \delta_{i j} \delta\left(\boldsymbol{r}-\boldsymbol{r}^{\prime}\right) \delta\left(t-t^{\prime}\right)$. As the complex Langevin trajectories are sampled, we compute operator averages by leveraging the ergodic principle.

\section{Species polarizabilities in field-theoretic simulations}

In our field-theoretic model, bead species $A$ is either $P B D$-like or $P A G E$-like and species $B$ is imidazole-like (Im-like). The PBD and PAGE backbones in the experimental section of this work have measured dielectric constants of $\epsilon_{P B D}=2.3$ and $\epsilon_{P A G E}=6.0$, as reported in Table 1. The dielectric constant of liquid 1-methylimidazole (MeIm) has been reported to be as large as $\epsilon_{\text {MeIm }} \approx$ $40 .{ }^{11}$ We note that fluid dielectric constants are typically sensitive to temperature, pressure, and other variables such as chain connectivity and addition of salt, so we do not attempt to achieve quantitative agreement with literature or reported values. Rather, we grant these beads molecular polarizabilities such that they have qualitatively correct emergent dielectric properties: most importantly, that $\epsilon_{P B D-l i k e}<\epsilon_{P A G E-\text { like }}<\epsilon_{\text {Im-like }}$ for pure fluid phases of the respective bead types, and that the dielectric constants have a reasonable magnitude when compared to the above reported and measured values. To estimate the emergent dielectric properties of the beads in our model, we can use the mean-field expression for the dielectric constant in the polarizable field theory

$$
\epsilon_{M F}=1+4 \pi \rho_{0} \alpha_{v}
$$

where $\alpha_{v}$ is the polarizability volume. We note that the true dielectric constant in field-theoretic simulations (FTS) will differ somewhat from the above mean-field expression, due to correlation 
effects that mean-field theory does not capture. In this work we use $\alpha_{v}^{(P B D-\text { like })}=0.011 b^{3}$, $\alpha_{v}^{(P A G E-\text { like })}=0.108 b^{3}$, and $\alpha_{v}^{(\text {Im-like })}=0.541 b^{3}$. Figure S22, below, shows the dielectric functions directly measured from FTS, for the PBD-like, PAGE-like and Im-like bead types, along with the corresponding mean-field dielectric functions. The pure-fluid dielectric constants, which correspond to the dielectric function at $k=0$, are roughly $\epsilon_{P B D-l i k e} \approx 2, \epsilon_{P A G E-l i k e} \approx 10$, and $\epsilon_{\text {Im-like }} \approx 47$.

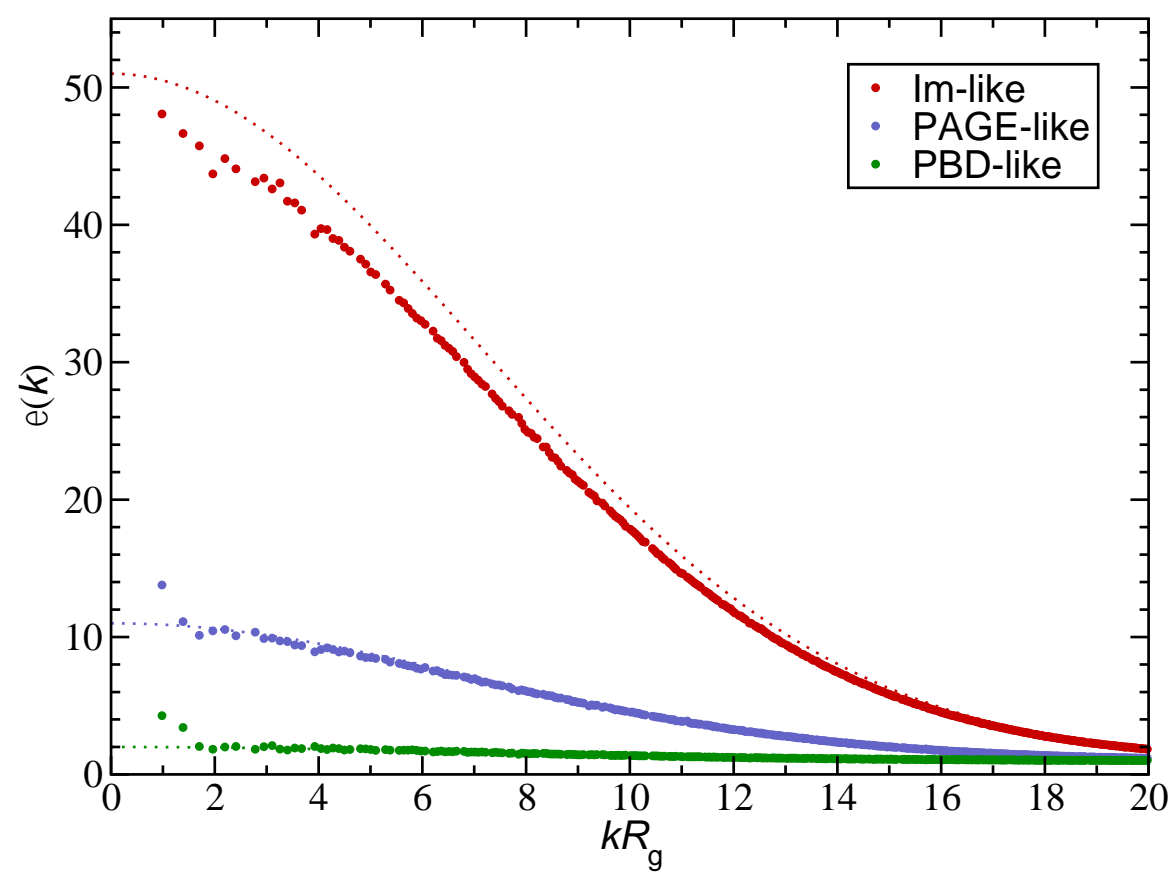

Figure S39. Dielectric function of a pure fluid phase of Im-like, PAGE-like and PBD-like monomers, at a density of $\rho_{0}=7.35 b^{-3}$, as measured in field-theoretic simulations. Dotted lines are the mean-field estimates.

\section{Numerical details of field-theoretic simulations}

The simulations presented in this work were conducted in a cubic box with dimensions $\left(6.4 R_{g}\right)^{3}$ and periodic boundary conditions. The spatial collocation mesh resolution was chosen to be $\Delta x=$ $a=0.1 R_{g}$, which is sufficient to resolve the smallest-scale features in our coarse-grained model. We solve the modified diffusion equations for the chain propagators using a pseudospectral operator-splitting approach with a chain contour resolution of $\Delta s=0.005 N_{b b}$. The CL equations of motion were propagated using the exponential time difference (ETD) algorithm with a time step $\Delta t=0.05$, and using mobility coefficients of unity for all $w$ fields and a mobility coefficient $\Lambda_{\varphi}=$ 20 which helps to accelerate thermalization of the electrostatic field. In all cases, the system is initialized in a random disordered configuration in which the fields have been equilibrated (this involves a warmup simulation on the order of $10^{6}$ timesteps). All simulations were performed on NVIDIA Tesla M2070, M2075, C2070, K80, P100 or V100 graphics processing units (GPUs). 
We use a low-variance structure factor operator, which is defined in Reference $11,{ }^{12}$ to compute the cation-cation structure factors $S_{++}(k)$. The structure factor at each state point in Figure 7 is the result of an average over between 3 and 5 independent field-theoretic simulations, each of which is initialized in an equilibrated, random disordered configuration, and thermally averaged for on the order of $10^{6}$ time-steps. The cation density calculations are the result of thermal averages of the cation density operator $\tilde{\rho}_{+}(\boldsymbol{r})$, which is given by

$$
\tilde{\rho}_{+}(\boldsymbol{r})=\frac{n_{+}}{V Q_{+}} e^{-\Omega_{+}(\boldsymbol{r})},
$$

and we perform the thermal average of this operator over $2 \times 10^{5}$ time-steps for both the PBDIm-like and PAGE-Im-like polymer to generate the cation density images in Figure 8.

\section{Anion-anion structure factor}

Figure S40 shows the comparison between the anion-anion structure factor and cation-cation structure factor for the PBD-like polymer.
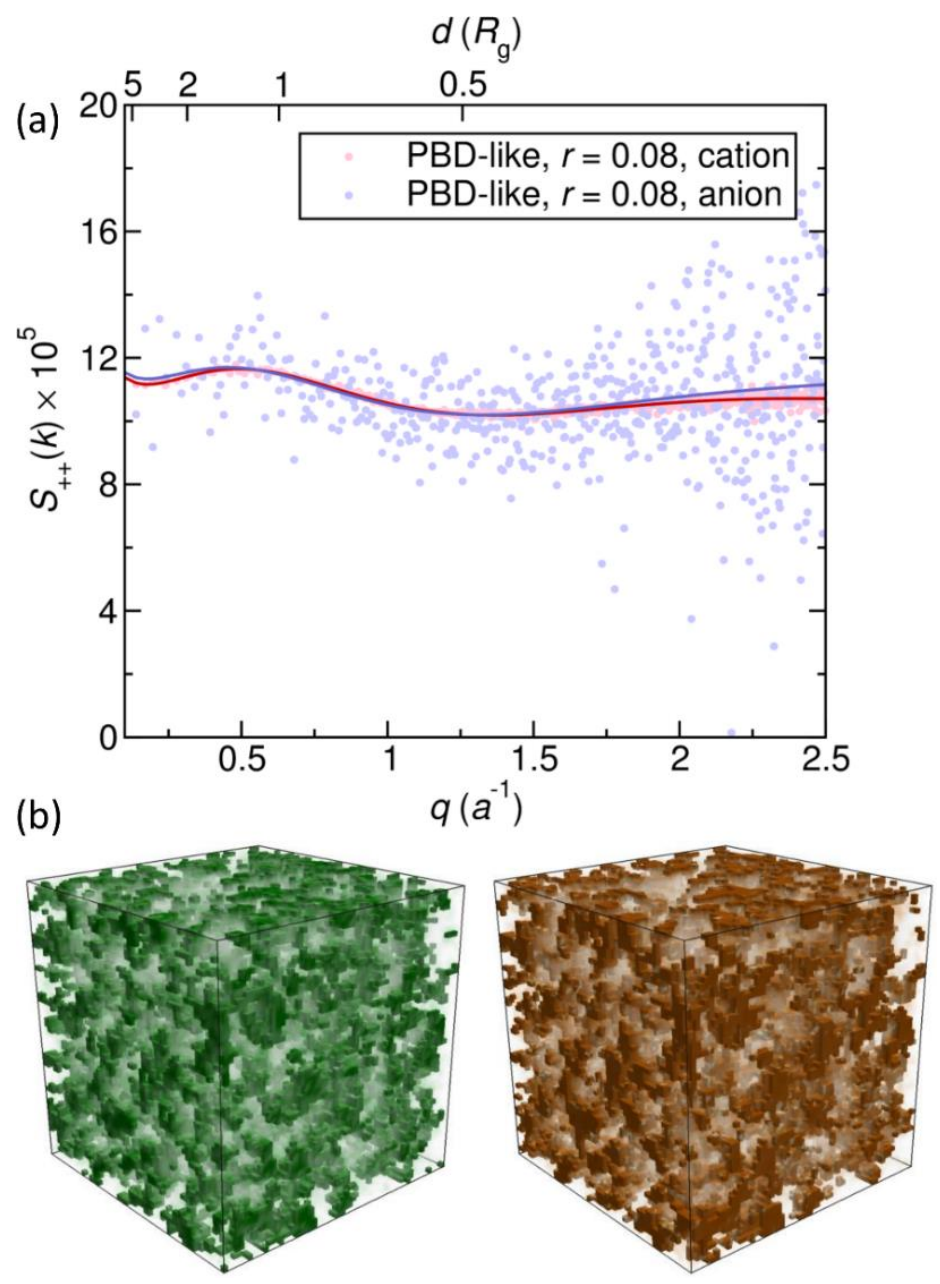

Figure S40. (a) Cation-cation and anion-anion structure factors show similar behavior with a peak indicating ion aggregation. (b) Ion channels, identified by the criterion $\phi_{+/-}(r) \geq\left\langle\phi_{+/-}\right\rangle \approx$ 0.014, form a percolating network for both cations and anions. 


\section{References}

1. Shane, J. J.; Höfer, P.; Reijerse, E. J.; de Boer, E., Hyperfine Sublevel Correlation Spectroscopy (HYSCORE ) of Disordered Solids. J. Magn. Reson. 1992, 99, 596-604.

2. $\quad$ McCracken, J.; Pember, S.; Benkovic, S. J.; Villafranca, J. J.; Miller, R. J.; Peisach, J., Electron Spin-Echo Studies of the Copper Binding Site in Phenylalanine Hydroxylase from Chromobacterium Violaceum. J. Am. Chem. Soc. 1988, 110, 1069-1074.

3. Jiang, F.; McCracken, J.; Peisach, J., Nuclear Quadrupole Interactions in Copper(II)Diethylenetriamine-Substituted Imidazole Complexes and in Copper(II) Proteins. J. Am. Chem. Soc. 1990, 112, 9035-9044.

4. Jiang, F.; Karlin, K. D.; Peisach, J., An Electron Spin Echo Envelope Modulation (ESEEM) Study of Electron-Nuclear Hyperfine and Nuclear Quadrupole Interactions of dz2 Ground State Copper(II) Complexes with Substituted Imidazoles. Inorg. Chem. 1993, 32, 25762582.

5. Kofman, V.; Farver, O.; Pecht, I.; Goldfarb, D., Two-Dimensional Pulsed EPR Spectroscopy of the Copper Protein Azurin. J. Am. Chem. Soc. 1996, 118, 1201-1206.

6. $\quad$ Grommen, R.; Manikandan, P.; Gao, Y.; Shane, T.; Shane, J. J.; Schoonheydt, R. A.; Weckhuysen, B. M.; Goldfarb, D., Geometry and Framework Interactions of ZeoliteEncapsulated Copper(II)-Histidine Complexes. J. Am. Chem. Soc. 2000, 122, 1148811496.

7. Taguchi, A. T.; O'Malley, P. J.; Wraight, C. A.; Dikanov, S. A., Hyperfine and Nuclear Quadrupole Tensors of Nitrogen Donors in the QA Site of Bacterial Reaction Centers: Correlation of the Histidine N $\delta$ Tensors with Hydrogen Bond Strength. J. Phys. Chem. B 2014, 118, 9225-9237.

8. Fredrickson, G. H., The Equilibrium Theory of Inhomogeneous Polymers. Oxford University Press: 2006.

9. Levi, A. E.; Lequieu, J.; Horne, J. D.; Bates, M. W.; Ren, J. M.; Delaney, K. T.; Fredrickson, G. H.; Bates, C. M., Miktoarm Stars via Grafting-Through Copolymerization: SelfAssembly and the Star-to-Bottlebrush Transition. Macromolecules 2019, 52, 1794-1802.

10. Panagiotou, E.; Delaney, K. T.; Fredrickson, G. H., Theoretical Prediction of an Isotropic to Nematic Phase Transition in Bottlebrush Homopolymer Melts. J. Chem. Phys. 2019, $151,094901$.

11. Zhang, H.; Geise, G. M., Modeling the Water Permeability and Water/Salt Selectivity Tradeoff in Polymer Membranes. J. Membrane Sci. 2016, 520, 790-800.

12. Düchs, D.; Delaney, K. T.; Fredrickson, G. H., A Multi-Species Exchange Model for Fully Fluctuating Polymer Field Theory Simulations. J. Chem. Phys. 2014, 141, 174103. 